\title{
The Trouble with Diffusion
}

\author{
R.T. DeHoff* and Nagraj Kulkarni \\ 166 Rhines Hall, University of Florida, Gainesville, Florida, USA
}

Received: September 27, 2001; Revised: July 10, 2002

\begin{abstract}
The phenomenological formalism, which yields Fick's Laws for diffusion in single phase multicomponent systems, is widely accepted as the basis for the mathematical description of diffusion. This paper focuses on problems associated with this formalism. This mode of description of the process is cumbersome, defining as it does matrices of interdiffusion coefficients (the central material properties) that require a large experimental investment for their evaluation in three component systems, and, indeed cannot be evaluated for systems with more than three components. It is also argued that the physical meaning of the numerical values of these properties with respect to the atom motions in the system remains unknown.

The attempt to understand the physical content of the diffusion coefficients in the phenomenological formalism has been the central fundamental problem in the theory of diffusion in crystalline alloys. The observation by Kirkendall that the crystal lattice moves during diffusion led Darken to develop the concept of intrinsic diffusion, i.e., atom motion relative to the crystal lattice. Darken and his successors sought to relate the diffusion coefficients computed for intrinsic fluxes to those obtained from the motion of radioactive tracers in chemically homogeneous samples which directly report the jump frequencies of the atoms as a function of composition and temperature. This theoretical connection between tracer, intrinsic and interdiffusion behavior would provide the basis for understanding the physical content of interdiffusion coefficients.

Definitive tests of the resulting theoretical connection have been carried out for a number of binary systems for which all three kinds of observations are available. In a number of systems predictions of intrinsic coefficients from tracer data do not agree with measured values although predictions of interdiffusion coefficients appear to give reasonable agreement. Thus, the complete connection has not been made, even for binary systems. The theory has never been tested in multicomponent systems.

An alternative path to understanding diffusion behavior in multicomponent systems is presented which is based upon a kinetically derived version of the flux equations. While this approach has problems of its own, it has the potential for providing a new range of insights into the process, and for devising simple models for predicting composition evolution in multicomponent systems.
\end{abstract}

Keywords: diffusion, formalism, simulation, jump frequency

\section{Introduction}

Diffusion must occur in any process that requires a change in local chemistry. In crystalline systems this requires that single atoms change their position on the crystal lattice. The average frequency with which this event occurs in a local volume element is different for each chemical component in the system, and varies with the composition and temperature of the volume element. The pattern of these events ultimately determines the evolution of the spatial distribution of the elements in a single phase crystal. Prediction and control of this chemical evolution requires an understanding of the diffusion process.

To achieve this understanding it is first necessary to be able to describe the diffusion process, i.e., to develop a formalism that relates how the atoms move to the current condition of the system. The phenomenological equations of

*e-mail: rdeho@mse.ufl.edu 
irreversible thermodynamics define fluxes as measures of the atom motions and relate them to forces defined in terms of gradients of the properties of the system calculated from the current condition of the system. These equations have been widely accepted as the basis for the description of the diffusion process.

Application of the phenomenological formalism to the description of interdiffusion of the elements in binary systems is straightforward and requires modest experimental effort. However, for three component systems, the formalism becomes cumbersome and the level of experimental effort required to evaluate the matrix of four interdiffusion coefficients defined for the system is significant. As the number of components in the system increases beyond three the number of diffusion coefficients needed to describe behavior increases rapidly. Further, values for the matrix of diffusivities in quaternary and higher order systems cannot be determined experimentally.

Evidently a practical description of diffusion behavior in multicomponent systems would require an ability to understand the physical content of these diffusion coefficients so that their values could be computed or at least estimated from more fundamental information. Tracer diffusion experiments, which report the penetration of a radioactive tracer of an element into a chemically homogeneous alloy, provide fundamental information about the frequency with which atoms of a component make a jump between adjacent sites on a crystal lattice. If the physical content of interdiffusion coefficients is to be understood, their values must be related to this fundamental information about the pattern of atom jump frequencies in the system.

Kirkendall's classic observation that the crystal lattice moves during diffusion first complicated then clarified this situation. Tracer information describes atom motion relative to the crystal lattice, but the Kirkendall observation showed that the crystal lattice moves in interdiffusion. Darken solved this problem by introducing the concept of intrinsic diffusion, describing motion of atoms in the nonuniform flowing system relative to the moving lattice.

The stage was set for what became the central theoretical problem in multicomponent diffusion: the determination of the connection between the fundamental tracer diffusion information, through the intrinsic diffusion coefficients to the practical interdiffusion coefficients. Without this theoretical connection the description of diffusion in multicomponent systems would remain impractical.

Subsequent sections review the experimental observables for each of the three kinds of diffusion experiments: tracer, intrinsic and interdiffusion. The formalisms devised to describe each of these kinds of experimental results are then recalled briefly, followed by a presentation of the established theoretical connection between the three kinds of information. Problems with the formalisms themselves are discussed. Experimental results that illuminate the inadequacies of the theory connecting these kinds observations are then presented. These deliberations support the thesis that the traditional phenomenological description of diffusion does not provide a useful or practical basis for collecting and organizing information about diffusion in a multicomponent system. An alternate approach to this problem is needed.

One such approach is based upon a kinetically derived flux equation which avoids the problems associated with the phenomenological approach. This equation is derived and discussed. In its rigorous version it has a potential for providing new insights into influences that operate in diffusion that bias the jumps of components relative to the diffusion direction. A simplified version has been shown to provide relatively simple models for diffusion behavior that successfully describe the experimental observables in a number of ternary systems. The latter has the potential to make the description of diffusion in multicomponent systems practical.

\section{Experimental Observables in Diffusion}

\section{Interdiffusion Experiments}

Diffusion produces changes in the distribution of chemical elements with time. The primary experiment designed to yield an understanding of diffusion behavior in an alloy system is based on the diffusion couple. Blocks of two alloys, $P$ and $Q$, are bonded at a temperature that is low enough so that the components in the alloys cannot intermix, Fig. 1. The distribution of each component is initially a step function, Fig. 1a. The couple is then taken to a high temperature and isothermally annealed for many hours or perhaps days. The components interpenetrate; each develops its composition profile after some time, t, Fig. 1b. The couple is cooled, sectioned and chemically analyzed to yield the functions $C_{\mathrm{k}}(\mathrm{x}, \mathrm{t}),(\mathrm{k}=1,2, \ldots, \mathrm{c})$ where $C_{\mathrm{k}}$ is the molar concentration (moles / cc) of component $\mathrm{k}$. The design of the couple guarantees that the flow of all of the components will be one dimensional (call it the $\mathrm{x}$ direction, perpendicular to the original bond interface). Generally the size of the two blocks is large compared to the diffusion zone (the zone of composition change) so that the boundary conditions for the diffusion process is "semi infinite"; this simply means that for sufficiently large values of $\mathrm{x}$ the composition profiles after the anneal remain at the original concentration values on each side of the couple.

The motion of the atoms of a component in such flowing systems is described by the concept of the interdiffusion flux of component $\mathrm{k}$. Focus upon a volume element $\mathrm{dV}$ in the couple, Fig. 2. Atoms of component $\mathrm{k}$ form a subset of all of the atoms in this volume. Define $v_{\mathrm{k}}$ to be the average velocity of $\mathrm{k}$ atoms in $\mathrm{dV}$ during some small time interval. 
Focus further upon the area element dA bounding the right side of $\mathrm{dV}$. The number of atoms of $\mathrm{k}$ that flow across $\mathrm{dA}$ reported per unit area and per unit time, is defined to be the flux of component $\mathrm{k}$ at dA. The area element is viewed as being at a fixed position in the couple relative to a coordinate system that is external to the couple. The flux of atoms relative to a value of $x$ that is fixed in the laboratory is called the interdiffusion flux of $\mathrm{k}$, can be shown to be $\mathrm{e}^{1-4}$

$$
J_{\mathrm{k}}{ }^{0}=C_{\mathrm{k}}\left(v_{\mathrm{k}}-\bar{u}\right)(k=1,2, \ldots, c)
$$

The superscript $\left(^{\circ}\right)$ in this and subsequent equations is used to describe properties associated with the interdiffusion process, i.e., with the motion of atoms in a fixed external (laboratory) reference frame. In this equation $\bar{u}$ is the molar
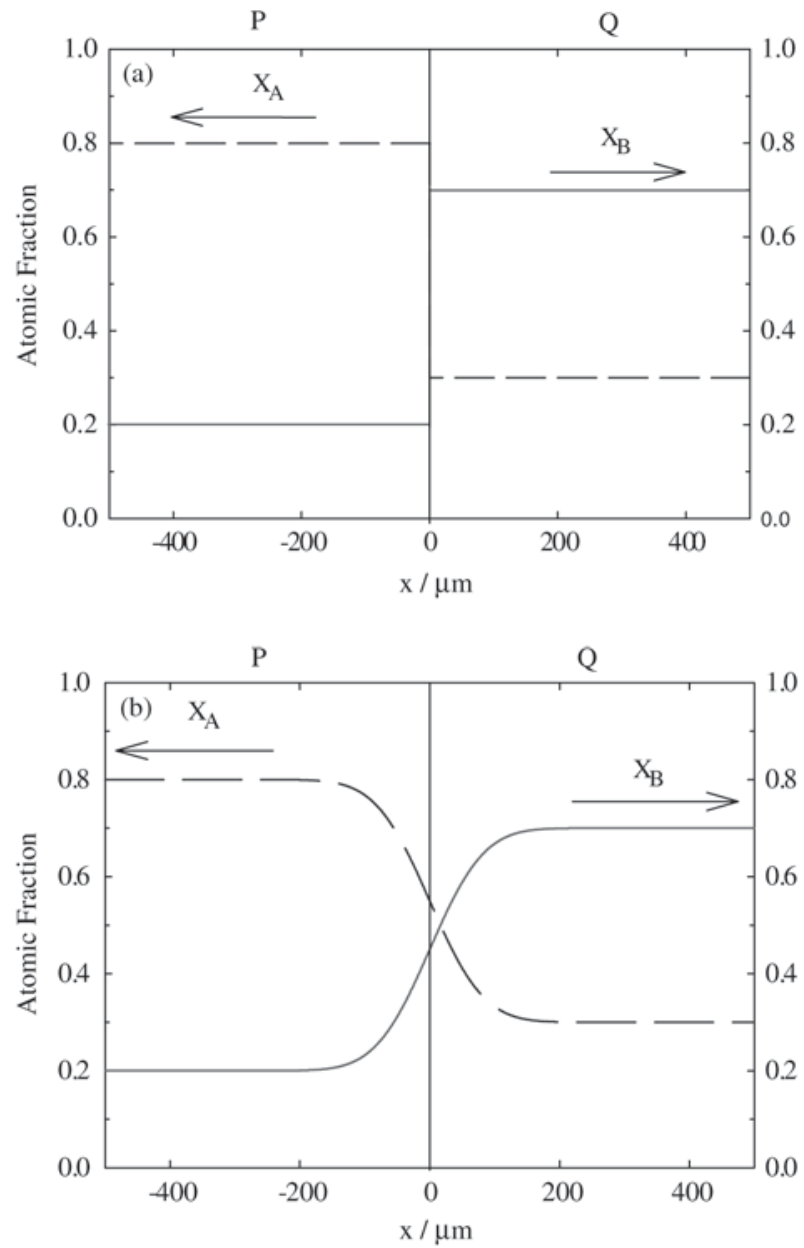

Figure 1. Composition distribution in a diffusion couple: a) before and b) after the isothermal anneal. An error function was employed to generate the composition profile where the interdiffusion coefficient, $\mathrm{D}^{\circ}=10^{-9} \mathrm{~cm}^{2} / \mathrm{s}$, and the time for the diffusion anneal, $\mathrm{t}=6 \mathrm{~h}$. average velocity (a "convective" velocity) of all of the components.

$$
\bar{u}=\sum_{k=1}^{c} X_{k} v_{k}
$$

$\mathrm{X}_{\mathrm{k}}$ is the atom fraction of component $\mathrm{k}$ in the volume element dV. It can be shown that this definition of the interdiffusion flux yields the relationship ${ }^{1-4}$

$$
\sum_{k=1}^{c} J_{k}^{o}=0
$$

That is, in a c component system there are (c - 1) independent interdiffusion fluxes. Dayananda ${ }^{5,6}$ showed that these fluxes can be evaluated from the concentration profile measurements without additional information:

$$
J_{k}^{o}(x)=\frac{1}{2 t} \int_{C_{k}-}^{C_{k}(x)} x d C_{k} \quad(k=1,2, \ldots, c)
$$

where $\mathrm{t}$ is the time of the diffusion anneal, $C_{\mathrm{k}}$ is the far field concentration of component $\mathrm{k}$ on the left side of the couple and $C_{\mathrm{k}}(\mathrm{x})$ is the concentration of component $\mathrm{k}$ at the position $\mathrm{x}$ in the couple. The derivation of this result neglects variations in molar volume in the system, a common assumption in diffusion theory. A sample result of this calculation is shown in Fig. 3. The concentration profiles for this demonstration were generated using ternary error function solutions given by Kirkaldy².

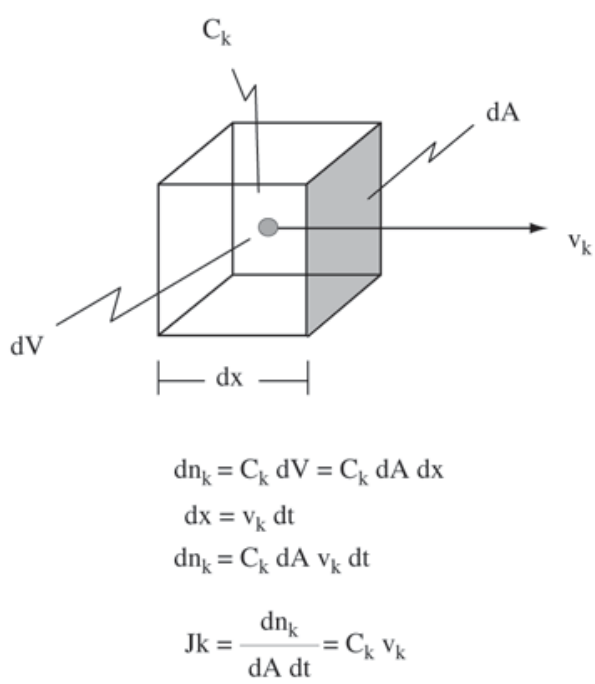

Figure 2. Interdiffusion flux in a flowing system. 

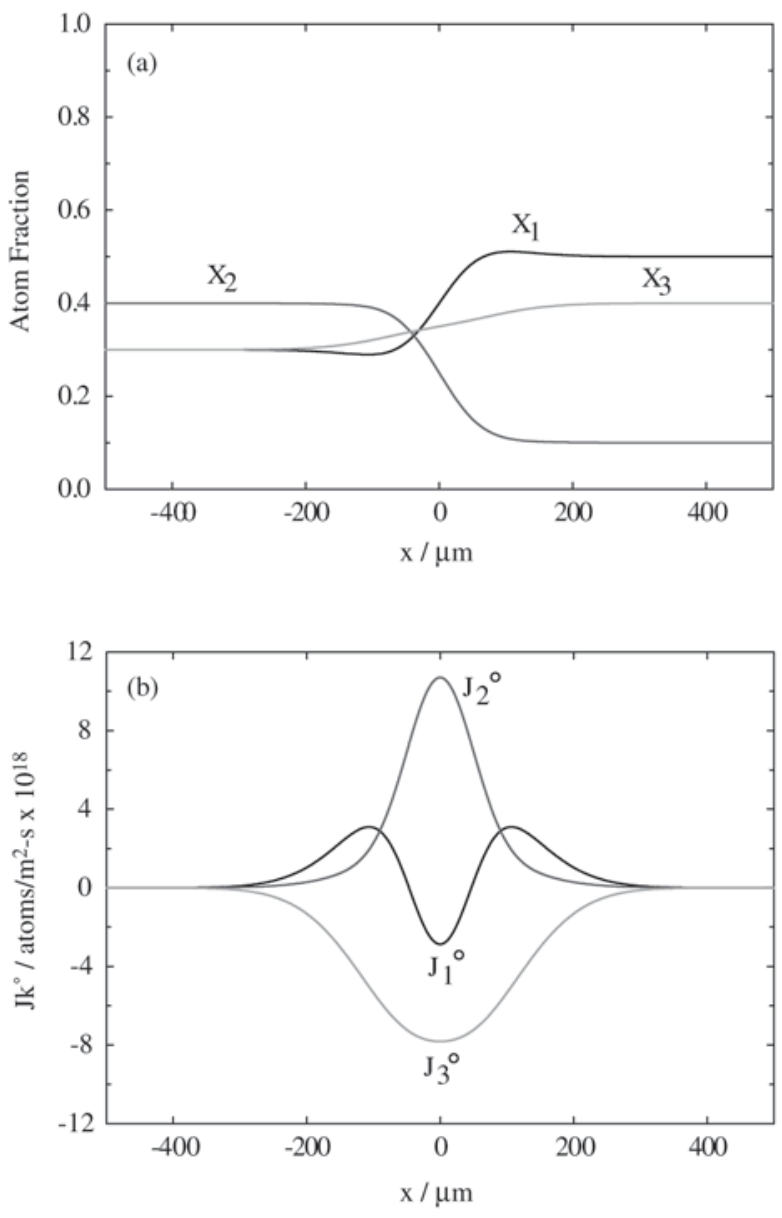

Figure 3. Calculation of the interdiffusion flux directly from the concentration profiles using Dayananda's equation ${ }^{5}$. a) concentration profiles; b) interdiffusion fluxes.

The typical interdiffusion experiment yields a pattern of composition profiles for all of the c-components in the system. The pattern of interdiffusion fluxes in that couple can be computed from this information for each of the components. Because these fluxes sum to zero, there are (c - 1) independent interdiffusion fluxes in a c-component system.

\section{Intrinsic Diffusion Experiments}

In 1947 Kirkendall and Smigelskas ${ }^{7}$ placed thin molybdenum wires at the original interface between two alloys $\mathrm{P}$ (pure $\mathrm{Cu}$ ) and $Q$ ( $\alpha$ brass, $\mathrm{Cu} 70 \% \mathrm{Zn} \mathrm{30 \% )} \mathrm{and} \mathrm{annealed}$ the resulting couple. The wires moved during the experiment, Fig. 4. Kirkendall concluded that the wires moved with the crystal lattice in the couple. Subsequent investigations established that this behavior is pervasive. The fundamental diffusion process in crystals is the motion of atoms from one lattice site to another, i.e., motion relative to the

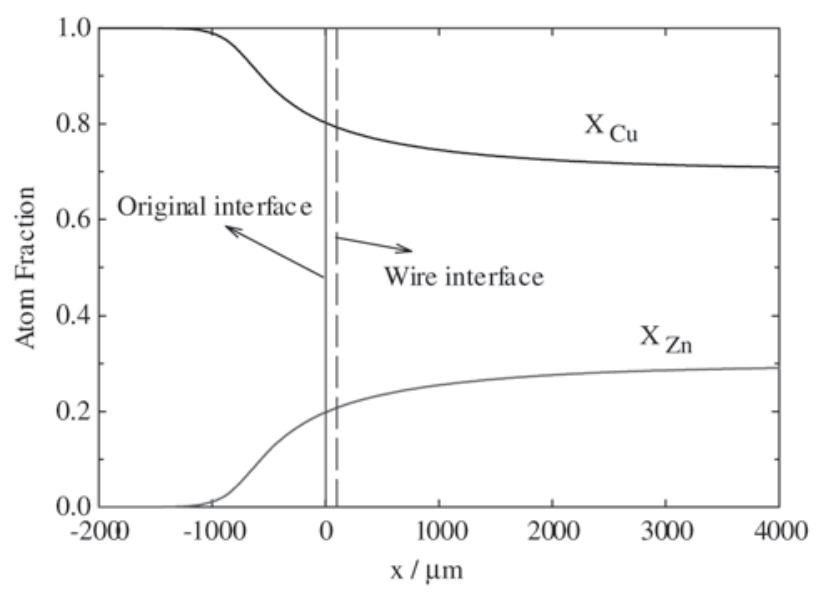

Figure 4. Displacement of wires in an interdiffusion experiment for the $\mathrm{Cu}-\mathrm{Zn}$ system, a phenomenon known as the Kirkendall effect $^{7}$.

crystal lattice. Kirkendall's experiment established that the crystal lattice is moving in the external fixed reference frame that is used to describe interdiffusion. Thus, in order to follow the fundamental diffusion process it is necessary to define the diffusion flux relative the moving crystal lattice:

$$
J_{\mathrm{k}}=C_{\mathrm{k}}\left(v_{\mathrm{k}}-v_{\mathrm{L}}\right)
$$

where $v_{\mathrm{L}}$ is the local lattice velocity. Darken ${ }^{8,9}$ defined this measure of atom motion relative to the moving lattice to be intrinsic diffusion. (In the notation of this paper intrinsic diffusion fluxes and other intrinsic properties have no superscript $\left(^{\circ}\right)$ in order to distinguished from the analogous interdiffusion properties.) A pervasive assumption in solid state diffusion is that the convective velocity $\bar{u}$ in equation (3) is negligible in the solid state so that the intrinsic and interdiffusion fluxes are related:

$$
J_{k}=J_{k}^{0}-C_{k} v_{L}
$$

Unlike the interdiffusion fluxes (equation (3)) all c intrinsic fluxes are independent. They sum, not to zero, but to the local value of the vacancy flux $J_{v}$ :

$$
\sum_{k=1}^{c} J_{k}=-J_{v}
$$

The original Kirkendall experiment and its successors could only provide the lattice velocity at the marker plane inherited from the original bond plane. Thus information on intrinsic diffusion could be obtained from a single composition in a given couple. In later years couples have been constructed with markers distributed through the diffusion 
zone ${ }^{3,10-14}$. Figure 5 shows one couple design that incorporates a marker plane that is oblique to the direction of diffusion. Upon sectioning after diffusion the markers have displaced forming a curve that may be described by a function, $\mathrm{g}(\mathrm{x})$. Philibert ${ }^{3,10,11}$ has devised an analysis that permits the determination of the lattice velocity distribution $v_{L}(\mathrm{x})$ from the marker pattern $\mathrm{g}(\mathrm{x})$ without simplifying assumptions. Thus, both terms in equation (6) are experimentally accessible and the intrinsic diffusion fluxes of all $\mathrm{c}$ components can be computed from experimental composition profiles and marker displacements without simplifying assumptions $^{12-14}$.

\section{Tracer Diffusion Experiments}

The classic tracer diffusion experiment begins with a block of an alloy of a desired composition. A thin layer containing the desired radioactive element is dispersed on one face of the block. The sample is annealed at a predetermined temperature for a predetermined time. The radioactive isotope permits measurement of the concentration of tracer as a function of depth of penetration even though the tracer element is present in very small quantity. Thus the
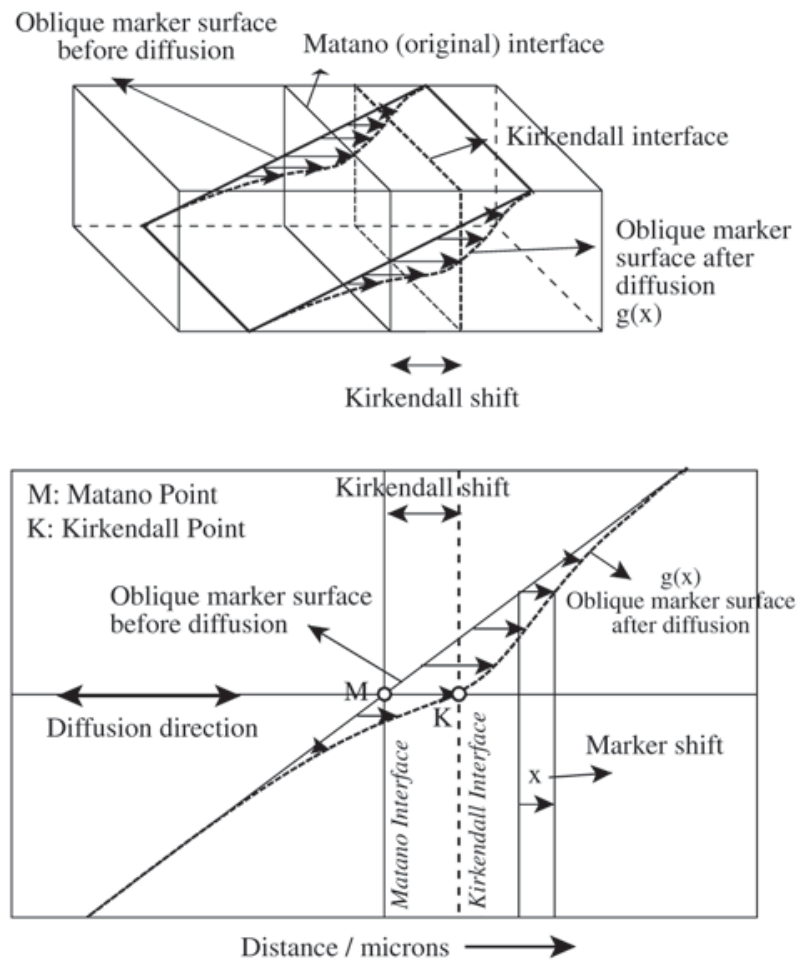

Figure 5. Oblique marker plane for the determination of lattice velocities for the entire diffusion zone before and after the interdiffusion anneal. experiment monitors the motion of one of the components into an alloy that is essentially chemically uniform. A straightforward analysis of the tracer profile yields the tracer diffusion coefficient, $D_{\mathrm{k}}{ }^{*}$, for that element in that alloy. A physical argument that describes the tracer flux in terms of the mean effective jump frequency of component, $\Gamma_{\mathrm{k}}{ }^{*}$, yields the relation ${ }^{2-4}$

$$
D_{k}^{*}=\frac{1}{6} \lambda^{2} \Gamma_{k}^{*}
$$

where $\lambda$ is the diffusion jump distance. The jump frequency for each component in a given system is found to depend upon the composition of the alloy and the temperature of the anneal. The number of jumps an atom makes between sites is the central physical descriptor of the diffusion process. In principle it would seem that, given the jump frequencies of each of the components as a function of composition one could predict the other experimental observables, i.e., the composition distribution function $\left(C_{\mathrm{k}}(\mathrm{x}, \mathrm{t})\right.$ for $\left.\mathrm{k}=1,2, \ldots, \mathrm{c}\right)$ and the lattice velocity distribution, $v_{\mathrm{L}}(\mathrm{x}, \mathrm{t})$ in a chemically evolving system. This connection between tracer, intrinsic and interdiffusion behavior is the focus of the physical theory of diffusion.

\section{Diffusion Formalism}

The information obtained from the diffusion experiments described above is explicit to that experiment. It is desirable to use that information for predicting diffusion behavior in other situations in the same alloy system. This has been achieved by devising a formalism that provides a general description of diffusion phenomena. The experimental results are then used to evaluate parameters introduced in the formal description. With the parameters evaluated for an alloy system, the general, formal equations may be used to predict diffusion behavior in that alloy system for any set of initial and boundary conditions.

The time evolution of the distribution of the compositions in a chemically evolving system is formally described by the phenomenological equations adapted from the thermodynamics of irreversible processes ${ }^{1-4,15-17}$. These equations are qualitatively based upon the notion that flow rates increase as a system gets farther from equilibrium. Flow rates are defined in terms of the fluxes of the things that move (quantity per $\mathrm{m}^{2}-\mathrm{sec}$ ) as in equation (1). The conditions for equilibrium specify that, in the absence of externally applied fields, gradients of certain local intensive thermodynamic properties are zero at equilibrium ${ }^{1,18-20}$, specifically,

$$
\begin{aligned}
& \operatorname{grad} T=0 \\
& \operatorname{grad} \mu_{k}=0 \quad(k=1,2, \ldots, c)
\end{aligned}
$$


where $\mu_{\mathrm{k}}$ is the chemical potential of component $\mathrm{k}$. In the absence of external fields in an isothermal system the chemical potential is an algebraic function of composition, so that the condition $\operatorname{grad} \mu_{\mathrm{k}}=0$ implies $\operatorname{grad} C_{k}=0$ $(\mathrm{k}=1,2, \ldots, \mathrm{c})$. When the gradients vanish, the corresponding flows are zero. It is natural to write the flow equation based on the assumption that each flux is proportional to all of the independent gradients in a nonequilibrium system.

\section{Interdiffusion Formalism}

For isothermal interdiffusion in a system with c components the phenomenological equations may be written

$$
J_{k}^{o}=-\sum_{j=1}^{c-1} L_{k j}^{o} \operatorname{grad} \mu_{j} \quad(k=1,2, \ldots, c-1)
$$

The components of the matrix of phenomenological coefficients, $\mathrm{L}_{\mathrm{kj}}{ }^{\circ}$, are called the mobilities in this formalism. It is these coefficients that must be evaluated from diffusion couple experiments. If they are determined as a function of composition and temperature then equation (10) permits prediction of the evolution of composition distribution in the system. Recall that, in a c-component system there are (c - 1) independent interdiffusion fluxes, equation (3).

An alternate, and more traditional formalism for describing the interdiffusion fluxes in a multicomponent system is a generalization of Fick's law for binary systems:

$$
J_{k}^{o}=-\sum_{j=1}^{c-1} D_{k j}^{o} \operatorname{grad} C_{j} \quad(k=1,2, \ldots, c-1)
$$

where $D_{\mathrm{kj}}{ }^{\circ}$ is a matrix of interdiffusion coefficients.

Note that in either formalism there are (c - 1) independent fluxes in the system (because the interdiffusion fluxes sum to zero, equation (3)) and (c - 1) terms in each equation. There are $(c-1)$ independent chemical potential gradients in equation (10) because the chemical potentials of the c components are related by the Gibbs-Duhem equation in thermodynamics ${ }^{1,2,18-20}$. There are (c - 1) independent concentration gradients in equations (11) because the atom fractions sum to 1 . There are thus $(\mathrm{c}-1)^{2}$ coefficients in the description of a c component system. In the thermodynamics of irreversible processes the principle or microscopic reversibility devised by Onsager ${ }^{16}$ shows that the square matrix of mobility coefficients is symmetrical, i.e. corresponding off-diagonal terms are equal. This reduces the number of independent phenomenological coefficients in a c-component system to $1 / 2 \mathrm{c}(\mathrm{c}-1)$. The two descriptions in equations (10) and (11) are interconvertible since the chemical potentials are functions of composition. Accordingly, the matrix of diffusion coefficients, $D_{\mathrm{kj}}{ }^{\circ}$, or, alternatively, the matrix of mobilities, $L_{\mathrm{kj}}{ }^{\circ}$, can be computed from one another ${ }^{2,3}$. The analyses of experimental penetration profiles yields the matrix of diffusivities ${ }^{2-4,21}$. If desired the matrix of mobilities can then be computed by combining the diffusivity results with a thermodynamic solution model that permits calculation of the chemical potentials from the concentrations.

\section{Intrinsic Diffusion Formalism}

Phenomenological equations used to describe intrinsic diffusion behavior appear to be very similar to equations (10) and (11) with two important differences:

a. The fluxes and diffusivities are "intrinsic" properties of the process, in the sense that this word is used in diffusion theory (indicated by dropping the superscript ${ }^{\circ}$ in the notation), and

b. The fluxes do not sum to zero, see equation (7).

For intrinsic diffusion:

$$
\begin{aligned}
& J_{k}=-\sum_{j=1}^{c-1} L_{k j} \operatorname{grad} \mu_{j} \quad(k=1,2, \ldots, c) \\
& J_{k}=-\sum_{j=1}^{c-1} D_{k j} \operatorname{grad} C_{j}(k=1,2, \ldots, c)
\end{aligned}
$$

There remain $(\mathrm{c}-1)$ terms in each flux equation (there remain $(\mathrm{c}-1)$ independent composition variables) but now there are $\mathrm{c}$ independent fluxes. The matrix of diffusion coefficients is thus not a square matrix, but contains $\mathrm{c}(\mathrm{c}-1)$ elements.

In principle the matrix of intrinsic diffusivities for a multicomponent system can be evaluated by combining experiments that report concentration evolution data and lattice marker displacement measurements. The corresponding matrix of mobilities can be computed from this information and a thermodynamic solution model to compute the chemical potentials.

\section{Problems with the Phenomenological Formalism and its Implementation}

\section{Interdiffusion}

The interdiffusion equations provide a basis for incorporating results of diffusion couple experiments into a framework that permits prediction of the evolution of concentration patterns for that system for any set of initial and boundary conditions. Given $C(\mathrm{x}, \mathrm{t})$ for a full range diffusion couple in a binary system, $D^{\circ}(C)$ can be computed from the classic Matano analysis, a procedure that has been in introductory textbooks for more than half a century ${ }^{1-4}$. Then, given $D^{\circ}(C)$ an appropriate simulation of 
the governing flux equation can be used to predict the chemical evolution for any other situation involving that binary alloy system.

The trouble begins when this analysis is extended to three component systems and beyond. In a three component system there are only two independent compositional variables and two independent interdiffusion fluxes. Thus, a variety flux equations can be written for the same system, depending upon which component is chosen to be the dependent variable. e.g.,

Component 3 as dependent variable:

$J_{2}^{0}=-D_{21}{ }^{30} \operatorname{grad} C_{1}-D_{22}{ }^{30} \operatorname{grad} C_{2}$

Component 1 as dependent variable:

$J_{2}^{0}=-D_{22}{ }^{10} \operatorname{grad} C_{2}-D_{23}{ }^{10} \operatorname{grad} C_{3}$

It becomes necessary to add a superscript to the notation to make explicit the choice of dependent variable made in writing the equation. It is evident that the coefficients $D_{22}{ }^{30}$ and $D_{22}{ }^{10}$, both of which report the effect of the gradient of component 2 on the flux of component 2, are not equal.

In general, the values of the phenomenological coefficients depend upon the choice of independent variable made in setting up the problem. In the light of this observation it is unlikely that these coefficients will convey much physical understanding of how the atoms move in the system. This approach may be used to describe the composition evolution, but not to explain or understand it.

The analysis can be significantly simplified if it is assumed that "off-diagonal terms", $D_{\mathrm{kj}}{ }^{\circ}$, which describe the contribution of the gradient of component $j$ to the flux of component $\mathrm{k}$, may be neglected as small in comparison with "on-diagonal terms", $D_{\mathrm{kk}}{ }^{\circ}$, which describes the contribution of gradient of component k upon its own flux, $J_{\mathrm{k}}$. This is a convenient and intuitive assumption because it decouples the flux equations and greatly simplifies the mathematics of the description. This is a dangerous assumption. Analysis of relations between coefficients defined for two different choices of dependent variable, as illustrated in equation (14) for example, shows that some off-diagonal terms in one description contain an on-diagonal term in the other description. For example it can be shown that $D_{32}{ }^{3}=D_{32}{ }^{1}-D_{33}{ }^{1}$. In addition one can always find local situations in the patterns that develop in which the concentration gradient for the on diagonal term is small so that the local flux is primarily determined by the off-diagonal term.

In order to determine the matrix of four diffusion coefficients in a three component system it is necessary to prepare and analyze two diffusion couples with composition paths that cross in the Gibbs triangle. At the position of the crossing point both couples will have the same composition (and hence the same set of values for the $D_{\mathrm{kj}}{ }^{\mathrm{o}}$ matrix) but different values of the fluxes and gradients. The two flux equations (11) for each couple yield four equations in four unknowns (the $D_{\mathrm{kj}}{ }^{\mathrm{o}}$ matrix), and may be solved for their values at that specific composition. To obtain the pattern of composition dependent functions for the diffusivities, $D_{\mathrm{kj}}{ }^{\circ}\left(C_{2}, C_{3}\right)$ for the whole system, it is necessary to construct and anneal a series of diffusion couples with composition paths that criss-cross each other in the Gibbs triangle. Values of the each of the four diffusivities are computed at crossing points. These isolated values may be analyzed statistically to deduce best fit functions, - four surfaces over the Gibbs triangle -, that complete the data base for interdiffusion in the system for the temperature chosen for the diffusion anneals. A complete data base would require that this process be repeated at other temperatures.

Because of the level of effort required to obtain these data there have been relatively few determinations of this matrix in the five decades since the procedures were first outlined $^{2}$. In their text, Kirkaldy and Young ${ }^{2}$ list 26 references reporting such analyses where "the data is sufficiently accurate and comprehensive that a closure with theory can be illustrated." Only four of these cover the full composition range or even an entire phase field. Evidently the level of effort required to generate interdiffusion data bases for three component alloys is difficult to justify. This situation is not helped by the fact that the interdiffusion coefficients do not convey a physical understanding of how the atoms move in the system as discussed above. The data do not contribute to a physical understanding of the processes going on in diffusion so that patterns might be divined or predictions hypothesized.

Determination of the matrix of nine diffusivities for a single composition point in a quaternary system requires an experiment involving a set of three diffusion couples with composition paths that intersect at a point in the three dimensional composition space of the Gibbs tetrahedron. Unfortunately the probability that three space curves intersect in three dimensional space constitutes a set of points of measure zero. Accordingly, this matrix has never been measured for any quaternary system*. Experimental methods for measuring interdiffusion coefficients in quaternary and higher order systems are evidently impractical.

\section{Intrinsic Diffusion}

Because the intrinsic diffusion fluxes are independent the description of the intrinsic behavior of a c component system requires an additional flux equation requiring addition of (c - 1) diffusion coefficients. Darken analyzed the problem of determining intrinsic diffusion coefficients in a binary system ${ }^{8-9}$. He derived the result

* The nine diffusivities have been estimated at a single quaternary composition in the Ni-Cr-Al-Mo system by constructing three incremental couples (i.e., with small composition differences) with crossing diffusion vectors and applying the approximation that the nine diffusion coefficients may be treated as constants in this composition interval ${ }^{22}$. 


$$
\begin{gathered}
D_{1}=D^{o}-\left(1-X_{2}\right)\left(\frac{\partial x}{\partial X_{2}}\right) v_{L} \\
D_{2}=D^{o}+X_{2}\left(\frac{\partial x}{\partial X_{2}}\right) v_{L}
\end{gathered}
$$

In a binary system the interdiffusion coefficient can be obtained by applying the Matano analysis to composition profiles and the lattice velocity can be obtained from marker displacements during diffusion. $D_{1}$ and $D_{2}$ may then be evaluated at any composition for which $v_{\mathrm{L}}$ is measured. The original Kirkendall experiment placed a marker at a single point, the original interface, and measured its displacement. Thus, $v_{\mathrm{L}}$, and hence $D_{1}$ and $D_{2}$, could be determined at a single composition in this experiment. That composition was not known a-priori; it was determined as the composition at the marker plane after the experiment. To generate a data base for a range of compositions required the preparation of a number of diffusion couples with initial composition pairs that produced marker plane compositions that spanned the range. If the oblique marker plane experiment, Fig. 5, is implemented, $v_{\mathrm{L}}$ can be determined at each composition in the couple. Combination with $D^{o}$ values for the couple will permit computation of the two intrinsic diffusivities at all compositions that exist in the couple. The values of $D_{1}$ and $\mathrm{D}_{2}$ are indicative of the relative rates of motions of the two components on the crystal lattice.

Darken and others extended his analysis to ternary systems ${ }^{2,9,23}$. Implementation of the analysis requires determination of the matrix of interdiffusion coefficients, $D_{\mathrm{kj}}{ }^{\circ}$, in the system using a pair of couples with intersecting composition paths with the attendant difficulties described above. In addition the lattice velocity must be determined in both couples at their common composition. Evidently this cannot be achieved with traditional, single marker couples because the compositions at the marker planes in each couple, where $\mathrm{v}_{\mathrm{L}}$ may be determined, will not in general coincide with the composition at the intersection point of their composition paths. The oblique marker plane experiment permits determination of $v_{\mathrm{L}}$ at all points in the compositions that exist in the couple, including that of the point of intersection of the composition paths. The resulting six equations (three flux equations in each couple) may be solved simultaneously to determine the matrix of six intrinsic diffusion coefficients at the composition of the intersection point.

Given the level of effort involved it is not surprising that intrinsic diffusivities have been estimated at but a few points in a few ternary systems ${ }^{24}$. There has been no systematic study attempting to determine this matrix, $D_{\mathrm{kj}}$, as a function of composition in any ternary system.
The extra effort might be justified in the light of the fact that intrinsic fluxes provide a more direct view of how the components move with respect to one another, since they report independent motions of the components relative to the crystal lattice. However this insight is not supplied by the numerical values of the elements of the diffusivity matrix, $D_{\mathrm{kj}}$. These properties suffer from the same problem cited for the interdiffusion coefficients: their values depend upon the choice of independent compositional variable in the analysis. $D_{22}{ }^{1}$ and $D_{22}{ }^{3}$ are different numbers, though both purport to describe the effect of the concentration gradient of component 2 on the intrinsic flux of component 2. Evidently the numerical values of the elements in the diffusivity matrix is not a useful basis for understanding these atom flows in the system.

The intrinsic fluxes themselves have the potential to provide a level of physical understanding of the real motion of the components in the system. Equation (6) provides the relationship between intrinsic fluxes and the interdiffusion fluxes and lattice velocities. On the right side of this equation Dayananda's analysis permits determination of the interdiffusion flux $J_{\mathrm{k}}{ }^{\circ}(\mathrm{x})$ for each component by applying equation (4) to the measured composition distribution. The lattice velocity distribution $v_{\mathrm{L}}(\mathrm{x})$ can be determined from a couple with an oblique marker plane, using Philibert's analysis. Thus the pattern of the intrinsic fluxes in a couple can be determined experimentally from the composition profiles and marker shift pattern in that couple. These relationships make no simplifying assumptions. Unfortunately, the oblique marker plane experiment has only been applied to a few binary systems ${ }^{25,26}$. It has never been applied to ternary or higher order systems, though the methodology makes such an application relatively straightforward.

\section{Summary of the Problems with the Phenomenological Formalism}

For the description of interdiffusion the implementation of the formalism requires the determination of $1 / 2 \mathrm{c}(\mathrm{c}-1)$ $D_{\mathrm{kj}}{ }^{\circ}$ values as functions of composition and temperature. This is straightforward for a binary system. A significant investment of experimental effort is required for a three component system. The approach cannot by implemented for quaternary and higher order systems. The description cannot be simplified by neglecting cross terms. The values obtained depend upon the choice of dependent compositional variable in setting up the description of the system. The pattern of values of the $D_{\mathrm{kj}}{ }^{\circ}$ 's for a system does not provide a basis for understanding the physics of the diffusion process.

The phenomenological description of intrinsic diffusion suffers from the same set of problems though the atom motions described are fundamental in the sense that the independent fluxes of the components relative to the lattice 
may be accessed in individual couples. Even more experimental effort is required to determine the matrix of diffusivities, since information on the pattern of motion of markers in the diffusion zone must be determined in experiments. This pattern has been measured on some binary systems ${ }^{12-14,24-26}$. It's measurement has not been undertaken in a single ternary system. Even if they were determined, the patterns of values of the $D_{\mathrm{kj}}$ matrix would not provide a basis for understanding the physic of the process.

\section{Connection Between Tracer, Intrinsic and Interdiffusion Information}

The phenomenological formalism was devised so that information extracted from diffusion couple experiments could be used to make more general calculations. It has proven to be a cumbersome basis for developing a diffusion data base. Perhaps if a physical understanding of the content of the diffusivities could be developed patterns could be recognized and correlations evoked that would foster the generation of a more useful diffusion data base. Tracer diffusion data provides the most direct link to a physical understanding of atom motions in binary and multicomponent systems. Darken recognized this in his initial paper on the subject $^{8}$. He set out to establish the connection between tracer diffusion information and intrinsic and interdiffusion behavior.

In proposing a solution to this problem Darken considered the phenomenological equations for tracer and intrinsic diffusion in a binary system:

Intrinsic Diffusion:
$J_{k}=-L_{k} \nabla \mu_{k}=-L_{k}\left(\frac{\partial \mu_{k}}{\partial C_{k}}\right) \nabla C_{k}=-D_{k} \nabla C_{k}$

Tracer Diffusion:
$J_{k}^{*}=-L_{k}^{*} \nabla \mu_{k}^{*}=-L_{k}^{*}\left(\frac{\partial \mu_{k}^{*}}{\partial C_{k}^{*}}\right) \nabla C_{k}^{*}=-D_{k}^{*} \nabla C_{k}^{*}$

where the superscript $\left(^{*}\right)$ indicates properties of the tracer experiment. The derivatives can be evaluated from solution thermodynamics ${ }^{2-4,8-9}$ :

$$
\begin{aligned}
& \underset{\text { Diffusion: }}{\text { Intrinsic }}\left(\frac{\partial \mu_{k}}{\partial C_{k}}\right)=\frac{k T}{C_{k}}\left(1+\frac{\partial \ln \gamma_{k}}{\partial \ln X_{k}}\right) \\
& \underset{\text { Diffusion: }}{\text { Tracer }}\left(\frac{\partial \mu_{k}^{*}}{\partial C_{k}^{*}}\right)=\frac{k T}{C_{k}^{*}}
\end{aligned}
$$

where $\gamma_{\mathrm{k}}$ is the activity coefficient of component $\mathrm{k}$ in the alloy system. Evaluation of the right hand side of the tracer equation is simplified because the tracer forms a very dilute solution in the otherwise uniform alloy. Darken then made a very plausible assumption: the mobility of a tracer in a uniform alloy is identical with the mobility of that component in a volume element in a flowing system that has the same composition and temperature:

$$
L_{\mathrm{k}}=L_{k}^{*}
$$

Inserting this assumption and the evaluation of the thermodynamic derivatives into equations (16) produces the Darken relation between tracer diffusivities and intrinsic diffusivities:

$$
D_{k}=D_{k}^{*}\left(1+\left(\frac{\partial \ln \gamma_{k}}{\partial \ln X_{k}}\right)\right) \quad(k=1,2)
$$

The predicted relation between interdiffusion coefficients and tracer behavior may then be derived:

$$
D^{o}=\left(X_{2} D_{1}^{*}+X_{1} D_{2}^{*}\right)\left(1+\left(\frac{\partial \ln \gamma_{k}}{\partial \ln X_{k}}\right)\right)
$$

In order to test Darken's hypothesis it is necessary to carry out all three kinds of diffusion experiments (tracer profiles, marker displacement and composition profile) over a composition range in a binary system. There are a few examples of binary systems in the literature for which this information exists. The definitive test combines tracer diffusion coefficients, thermodynamic information and intrinsic diffusivity measurements to test equations (19). Analyses for a number of these systems have been reported in the literature ${ }^{12-14,25-31}$. If the two components have similar tracer behavior then the tests support Darken's hypothesis within experimental error ${ }^{14}$. However if the tracer behavior is significantly different, then the results show that the theory is inadequate to explain the differences between tracer and intrinsic diffusivities. Examples of tests for such systems are shown in Figs. 6 and 7. Assessments of the data have shown that the disagreement in the intrinsic diffusion coefficients and hence the lattice shifts, are well outside the experimental error in these systems. It must be concluded from these results that Darken's hypothesis yields a theory that is inadequate to explain the connection between tracer and intrinsic diffusion coefficients.

Manning ${ }^{23,36-39}$ and others ${ }^{17,28,40}$ have analyzed this connection at a more sophisticated level, introducing concepts such as the vacancy wind effect. Examples of the predictions of Manning's more detailed analysis are also shown 

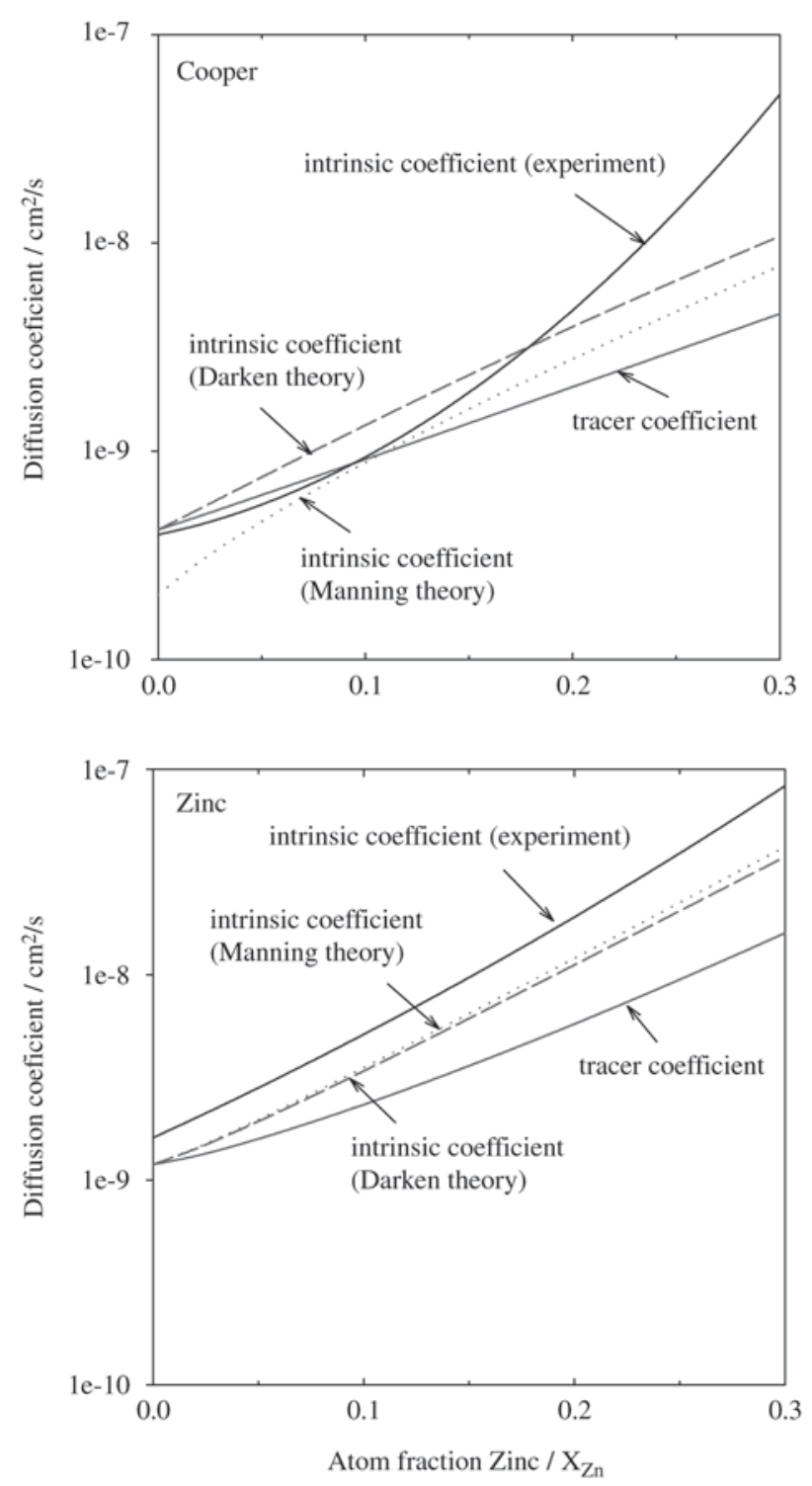

Figure 6. Darken-Manning relations in the $\mathrm{Cu}-\mathrm{Zn}$ system at $915^{\circ} \mathrm{C}$. The experimental intrinsic diffusion data was obtained from Horne $\& \mathrm{Mehl}^{32}$, the tracer data was obtained from Anusavice \& DeHoff ${ }^{33}$ and the thermodynamic data was obtained from Kowalski and Spencer ${ }^{34}$.

in Figs. 6 and 7. In some cases the additional term produces a small correction to Darken's prediction, within the range of experimental error in most cases. In other cases Manning's correction is in the wrong direction.

Darken's treatment, and Manning's corrections, have

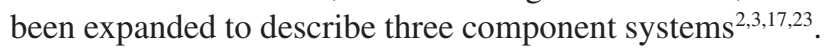
Experimental studies in a few ternary systems would provide a truly definitive test of this explanation of the
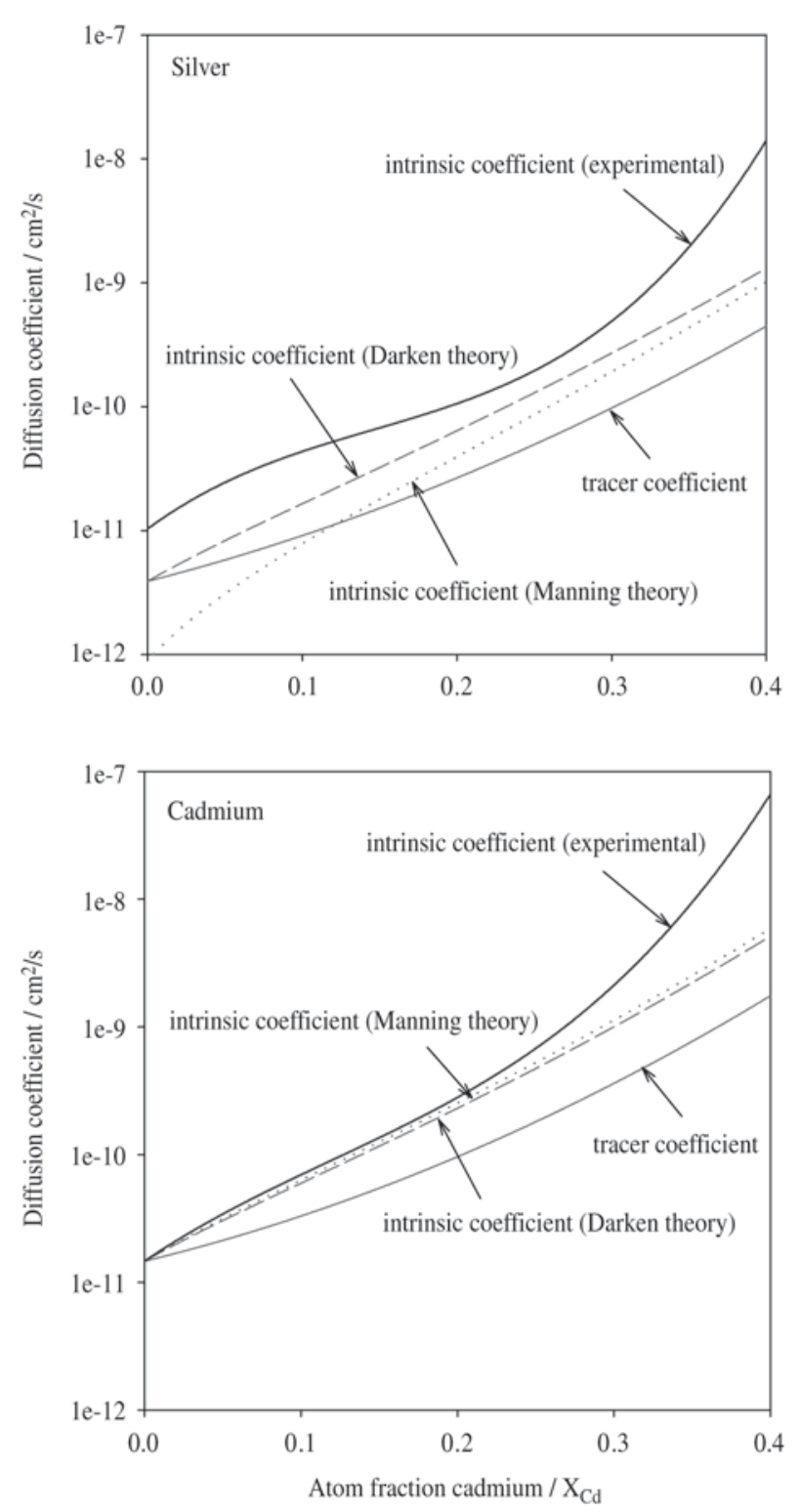

Figure 7. Darken-Manning relations in the Ag-Cd system at $600{ }^{\circ} \mathrm{C}$. The experimental intrinsic diffusion and tracer diffusion data were obtained from Iorio et al. ${ }^{29}$ and the thermodynamic data was obtained from Filby and Pratt ${ }^{35}$.

phenomenological coefficients. The effort involved to make such a series of tests appears daunting. There is but one ternary system for which tracer data exists for all three components over a significant composition range: $\mathrm{Cu}-\mathrm{Ni}-\mathrm{Zn}^{33}$. Interdiffusion data exists for the same system in the same composition range. There is anecdotal information about marker displacements for couples in this system, but no systematic study exists that would permit determination of the intrinsic diffusion coefficients. It is possible that, with 
the use of a suitable simulation of intrinsic diffusion one could arrive at an intrinsic diffusion model that provides a satisfactory description of the available marker data, but this has not been reported. Thus, the generalization of Darken's hypothesis to ternary systems has not been tested.

The phenomenological formalism is too cumbersome to be practical, and too abstruse to be physically meaningful. Hypotheses designed to provide physical interpretation of the descriptors of diffusion have proven to be inadequate. The formalism has not provided the physical context needed to explain or understand observed diffusion behavior. A diffusion data base without such a context is severely limited. The science of diffusion behavior is ripe for an alternate point of view.

\section{Jump Frequency Kinetic Formalism}

In parallel with the evolution of the phenomenological formalism there has been a continuing development of kinetic descriptions of diffusion processes. This approach seeks to devise flux equations for the components beginning with the jump frequency of the atoms. Early applications of this point of view established connections between tracer diffusion coefficients and the jump frequency of the tracer atoms. These studies identified correlation effects in self diffusion ${ }^{26,36-39}$ and demonstrated the complexities of behavior even in dilute binary and ternary alloys ${ }^{2-4}$. Applications of these descriptions to concentrated binary solutions, much less multicomponent systems, appears to require a level of detailed information about the system that is not experimentally accessible.

A version of this kinetic approach has been developed

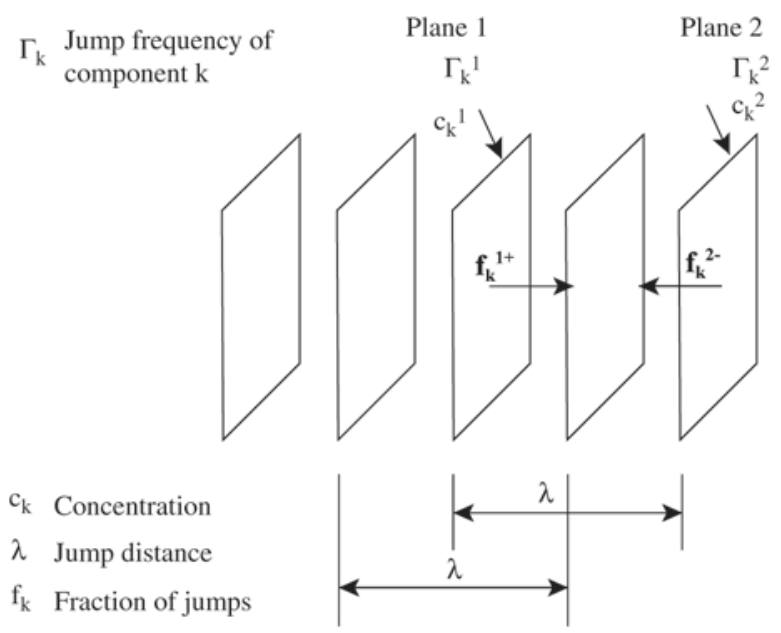

Figure 8. Schematic representation of two adjacent planes in a solid, emphasizing the diference in the concentration and jump frequency of a particular species upon each of the planes. at the University of Florida, along with a simulation of diffusion behavior that permits modeling and prediction of patterns of behavior of the experimental observables. This theory begins with the assumption that the jump frequencies of the components in a multicomponent diffusion process are functions only of the local thermodynamic state, (i.e., functions of composition in an isothermal system). This hypothesis is analogous to the assumption in the phenomenological approach that the individual diffusivities or mobilities are functions only of the local composition.

Consider two adjacent lattice planes, labeled 1 and 2, a distance $\lambda$ apart; $\lambda$ is the jump distance in the diffusion formulation, Fig. 8. Define $\Gamma_{\mathrm{k}}$ to be the jump frequency of atoms of component $k$ on plane 1. In an isothermal diffusion couple $\Gamma_{k}$ is a function of composition, and therefore position in the system. $\Gamma_{\mathrm{k}} \mathrm{dt}$ is the average number of jumps made by an atom of component $k$ on for the composition on plane 1 in a time interval dt. Let $\mathrm{C}_{\mathrm{k}}$ be the molar concentration of atoms of component $k$ on plane 1. Approximate the variation of these quantities with position as continuous functions. Then their values on the two planes are

$$
\begin{aligned}
& C_{\mathrm{k}} \text { (plane 1) } \quad C_{k}+\lambda \frac{d C_{k}}{d x} \text { (Plane 2) } \\
& \Gamma_{\mathrm{k}} \text { (plane 1) } \quad \Gamma_{k}+\lambda \frac{d \Gamma_{k}}{d x} \text { (Plane 2) }
\end{aligned}
$$

Let dA be the area of each of the planes. The volume occupied by the atoms on these planes is $\lambda$. The number of atoms of k in the slab associated with plane 1 is $C_{\mathrm{k}} \times \lambda \mathrm{dA}$. On the average each atom of $\mathrm{k}$ makes $\Gamma_{\mathrm{k}} \mathrm{dt}$ jumps in a time interval dt. The total number of jumps made by the atoms of component k on plane 1 in time $\mathrm{dt}$ is $\left(C_{\mathrm{k}} \times \lambda \mathrm{dA}\right) \times\left(\Gamma_{\mathrm{k}} \mathrm{dt}\right)$. Let $f_{\mathrm{k}+}$ be the fraction of these atoms jumps that take the atom of $\mathrm{k}$ to plane 2 . The number of $\mathrm{k}$ atoms transferred from plane 1 to plane 2 in time dt is

$$
d n_{\mathrm{k}, 1-2}=f_{\mathrm{k}+}\left(C_{k} \lambda \mathrm{dA}\right)\left(\Gamma_{\mathrm{k}} \mathrm{dt}\right)
$$

In an isotropic crystal, in the absence of bias effects in the jumping process, $f_{\mathrm{k}+}$ would be $1 / 6$.

This analysis can also be applied to atoms on plane 2 that jump to plane 1 using values for composition and jump frequency for $\mathrm{k}$ on plane 2 . The total number of atoms of $\mathrm{k}$ on plane 2 that make a jump to plane 1 in time $\mathrm{dt}$ is

$d n_{k, 1-2}=f_{k-}\left(C_{k}+\lambda \frac{d C_{k}}{d x}\right) \lambda d A \cdot\left(\Gamma_{k}+\lambda \frac{d \Gamma_{k}}{d x}\right) d t$

where $f_{\mathrm{k}-\mathrm{s}}$ is the fraction of atom jumps of $\mathrm{k}$ on plane 2 that move the atom to plane 1 . To obtain the net number of 
atoms of $\mathrm{k}$ that transferred from plane 1 to plane 2 in time dt subtract the number that jump from 2 to 1 from the number that jump from 1 to 2 .

$$
\begin{gathered}
d n_{k n e t}=d n_{k, 1-2}-d n_{k, 2-1} \\
d n_{k n e t}=f_{k+} C_{k} \lambda d A \Gamma_{k} d t-f_{k-}\left(C_{k}+\lambda \frac{d C_{k}}{d x}\right) \lambda d A\left(\Gamma_{k}+\lambda \frac{d \Gamma_{k}}{d x}\right) d t
\end{gathered}
$$

Multiply the factors on the right side of the equation and neglect the term involving $\lambda^{3}$. Collect terms and simplify:

$d n_{k n e t}=\left[\left(f_{k^{+}}-f_{k^{-}}\right) \lambda C_{k} \Gamma_{k}-f_{k^{-}} \lambda^{2} \frac{d C_{k} \Gamma_{k}}{d x}\right] d A d t$

The flux of component $\mathrm{k}$ from plane 1 to plane 2 is the number of atoms transferred per unit area per unit time:

$J_{k}=\frac{d n_{k n e t}}{d A d t}=\left(f_{k^{+}}-f_{k^{-}}\right) \lambda C_{k} \Gamma_{k}-f_{k^{-}} \lambda^{2} \frac{d C_{k} \Gamma_{k}}{d x}$

This is a kinetic equation for the intrinsic flux of component $\mathrm{k}$ (as contrasted to a phenomenological equation). All of the terms in the equation have a defined physical meaning. Note that this equation applies to component $\mathrm{k}$ no matter how many components there are in the system.

The first term in this equation contains the factor $\left(f_{\mathrm{k}+}-f_{\mathrm{k}-}\right)$. If the jumps between plane 1 and plane 2 are not biased by the flow process, then $f_{\mathrm{k}+}=f_{\mathrm{k}-}$, and the first term in equation (26) is zero. If this term is not zero, then influences are operating to produce a bias in the directions of jumps for atoms of component k. Accordingly the first term in equation (26) may be defined as a biased component to the intrinsic diffusion flux:

$$
J_{\mathrm{k}}^{\mathrm{B}}=\left(J_{\mathrm{k}+}-J_{\mathrm{k}-}\right) \lambda C_{\mathrm{k}} \Gamma_{\mathrm{k}}
$$

Presumably this bias is associated with the fact that there is a direction to the net flow of atoms in the system.

These fractions may be expressed in terms of their deviation from the isotropic values of $1 / 6$ :

$$
f_{k^{+}}=\frac{1}{6}+\alpha_{k^{+}} \text {and } f_{k^{-}}=\frac{1}{6}+\alpha_{k^{-}}
$$

On a given plane the fractions of jumps in all six direction sum to 1 . If it is assumed that the bias in jumps in the direction of diffusion does not affect the jumps occurring perpendicular to the flow direction

$$
1=\frac{1}{6}+\frac{1}{6}+\frac{1}{6}+\frac{1}{6}+f_{k^{+}}+f_{k^{-}}=\frac{2}{3}+\left(\frac{1}{6}+\alpha_{k+}\right)+\left(\frac{1}{6}+\alpha_{k^{-}}\right)
$$

This yields the result that

$$
\alpha_{\mathrm{k}+}=-\alpha_{\mathrm{k}-}
$$

That is the deviation from the isotropic values for the fractions for jumps in the direction of the flux and against it are equal in magnitude and opposite in sign. The difference

$$
f_{\mathrm{k}}-f_{\mathrm{k}-}=\alpha_{\mathrm{k}+}-\alpha_{\mathrm{k}-}=\alpha_{\mathrm{k}+}-\left(-\alpha_{\mathrm{k}+}\right)=2 \alpha_{\mathrm{k}+}
$$

The sign and magnitude of $\alpha_{k+}$ varies with position in a couple and is a different function for each component.

Since the net number of atoms transferred is a very small fraction of the total number of atoms that jump it may be argued that $\alpha_{k+}$ is very small in comparison to $1 / 6$. Thus, in the second term in equation (26) $f_{\mathrm{k}-}$ may be replaced by $1 / 6$. This term may be written

$$
J_{k}^{U}=-\frac{1}{6} \lambda^{2} \frac{\partial C_{k} \Gamma_{k}}{\partial x}
$$

This is the value of the intrinsic flux if the biased term is zero and may thus be defined as the unbiased contribution to the intrinsic vacancy flux.

With these evaluations the total flux of component $\mathrm{k}$ may be rewritten

$$
J_{k}=J_{k}^{B}+J_{k}^{U}=2 \alpha_{k^{+}} \lambda C_{k} \Gamma_{k}-\frac{1}{6} \lambda^{2} \frac{\partial C_{k} \Gamma_{k}}{\partial x}
$$

This is a kinetic flux equation, based upon a physical description of the atomic processes involved.

The product $\left(C_{\mathrm{k}} \Gamma_{\mathrm{k}}\right)$ has the units (jumps of $\mathrm{k}$ atoms per $\mathrm{m}^{3}$ per second) and may be visualized as the local concentration of atom jumps of $k$ per second. This yields the physically visualizable and plausible result that the unbiased flux component is determined by the gradient of the local concentration of atom jumps of $\mathrm{k}$ per second.

The biased component of the flux is introduced in this formalism. The role that it plays in the observable flux, and the influences that determine the value of $\alpha_{k+}$, are yet to be explored.

There is no phenomenological assumption in this equation. This does not mean that equation (33) violates the tenet of irreversible thermodynamics that states that each flux is influenced by all of the driving forces, equations (10) and (11). Because the jump frequency of component $\mathrm{k}$ depends upon all of the (c - 1) concentration variables the gradient term in equation (33) implicitly contains all of the concentration gradients. It is likely that $\alpha_{\mathrm{k}+}$ also depends on the magnitudes of the flows in the system, and thus all of the independent gradients.

Equation (33) describes the flux of component $\mathrm{k}$ no matter how many other components there are in the system. 
It is a flux equation that applies to multicomponent systems.

The number of descriptors in this equation, $\Gamma_{k}\left(C_{k}\right)$ and $\alpha_{k+}$, is much smaller than the $1 / 2 \mathrm{c}(\mathrm{c}-1)$ descriptors in equations (10) and (11). However, this simplification is mitigated by the fact that the content of $\alpha_{k+}$ is as yet unknown. In a sense, all of the complexity of the kinetic approach is contained in $\alpha_{k+}$ in the biased term. The challenge posed by this formalism is to develop an understanding of what influences operate to bias the jump direction of atoms, and how these influences operate.

\section{Simulation of Diffusion Behavior}

The central fundamental problem in the description of diffusion is the explanation of the connection between the three classes of experimental observables. The penetration of tracers in uniform alloys, the pattern of lattice velocities, and the evolution of the concentration distribution, are the observables derived from tracer, intrinsic and interdiffusion experiments. The corresponding data bases are sets of tracer, intrinsic and interdiffusion coefficients. In order to explore the connection between these data sets it is essential to be able to convert data base information to the corresponding experimental observables so that comparisons can be made. A flexible computer simulation of diffusion behavior that can take any of the three types of data base information and predict the corresponding experimental observable would be a very useful analytical tool. The same simulation could be used to explore the terms in the kinetic formalism.

\section{Simulation of Interdiffusion}

The data base is the set of interdiffusion coefficients, $D_{\mathrm{kj}}{ }^{\circ}$, as a function of all of the composition variables in a multicomponent system. The experimental observables are the composition profiles, $C_{\mathrm{k}}(\mathrm{x}, \mathrm{t})$ for th set of c components. These are connected by the interdiffusion flux equation (11). The simulation focuses on a slab-shaped volume element of thickness $\mathrm{dx}$ at position $\mathrm{x}_{\mathrm{i}}$ in the couple. In a given time step the change in the number of atoms of component $\mathrm{k}$ in that slab may be computed by applying a finite difference version of equation (11) to evaluate the interdiffusion flux of $k$ at $x_{i}$ and $x_{i}+d x=x_{i+1}$, positions that are fixed in the external reference frame. This calculation can be applied to each of the (c - 1) independent components. The fact that the fluxes in the laboratory frame sum to zero guarantees that there will be no change in the total number of atoms in the volume element between $x_{i}$ and $x_{i+1}$. New values of concentration of each component in the volume element at the end of the time step may be computed and assigned to the midpoint of the element. Values of the concentrations at $\mathrm{x}_{\mathrm{i}}$, $\mathrm{x}_{\mathrm{i}+1}$, etc. are interpolated to provide the starting concentration distribution for the next time step. Time steps are re- peated until a stable pattern of composition profiles (normalized by plotting versus $x / \sqrt{t}$ ) is obtained. The results may be compared with experimentally determined composition profiles, or with the experimental pattern of paths in composition space. Since these connections only involve definitions there are no hypotheses to be tested. In this application the simulation provides a valuable assessment of the consistency of interdiffusion data.

\section{Simulation of Intrinsic Diffusion}

The data base is the set of intrinsic diffusion coefficients, $D_{\mathrm{kj}}$, as functions of composition. Besides the concentration distribution, $C_{\mathrm{k}}(\mathrm{x}, \mathrm{t})$, the additional experimental observable is the distribution of lattice velocities, $v_{\mathrm{L}}(\mathrm{x}, \mathrm{t})$ which characterizes intrinsic diffusion. The connection between the data base and the observables is the phenomenological equations for intrinsic diffusion, equations (13). In the lattice (intrinsic) reference frame the $\mathrm{c}$ intrinsic diffusion fluxes are independent. Focus on a slab of thickness $\mathrm{dx}_{\mathrm{i}}$ at position $x_{i}$ at some time $t$. In the next time step use a finite difference version of equation (13) to compute the intrinsic fluxes of component $\mathrm{k}$ at $\mathrm{x}_{\mathrm{i}}$ and at $\mathrm{x}_{\mathrm{i}}+\mathrm{dx}_{\mathrm{i}}$. This permits the computation of the change in the number of atoms of $\mathrm{k}$ in $\mathrm{dx}_{\mathrm{i}}$ during that time step.

Because all $\mathrm{c}$ fluxes are independent the total number of atoms of all of the components in $\mathrm{dx}_{\mathrm{i}}$ will in general change during the time step. Some slab elements will finish the time step at $\mathrm{t}+\mathrm{dt}$ with more atoms than were there at time $\mathrm{t}$, and some will have less. These accumulations are accommodated by changing the thickness of each slab by an appropriate value, $\delta \mathrm{dx}_{\mathrm{i}}$, which will preserve the average volume of atoms in the element*. The position of each slab boundary at time $t, x_{i}(t)$, translates to some new position $x_{i}(t+d t)$ which is the position at $\mathrm{x}_{\mathrm{i}}(\mathrm{t})$ plus the sum of all of the slab thickness changes $\delta \mathrm{dx}_{\mathrm{i}}$ for all of the slabs from the left end of the diffusion couple to $\mathrm{x}_{\mathrm{i}}(\mathrm{t})$.

$$
x_{i}(t+d t)=x_{i}(t)+\sum_{j=0}^{i-1} \delta d x_{j}
$$

Since the total number of atoms of $\mathrm{k}$ and the total number of atoms in the slab are computed in each slab, the value of the atom fraction of $\mathrm{k}$, and hence the molar concentration $C_{\mathrm{k}}$, can be computed in each volume element at the end of the time step. An updated composition distribution may then be described at the end of the time step. The shift in position of each value of $x_{i}(t)$ corresponds to the incremental change in the position of a marker that may have existed at $x_{i}$; the local velocity of a marker at $x_{i}(t)$ may be computed as

$$
v_{L}\left(x_{i}(t)\right)=\frac{x_{i}(t+d t)-x_{i}(t)}{d t}
$$

\footnotetext{
* Inclusions of the variation of molar volume with composition in the simulation appears to be relatively straightforward, but has not been undertaken at this writing.
} 
Iterations of the time step are continued until stable distributions in a plot of $C_{\mathrm{k}}(\mathrm{x}, \mathrm{t})$ and $v_{\mathrm{L}}(\mathrm{x}, \mathrm{t})$ are obtained in $x / \sqrt{t}$ coordinates.

The simulation provides definitive tests for the formalism and the consistency of the data for binary systems for which $D_{1}$ and $D_{2}$ are available. The oblique marker plane experiment has been used only in a few isolated cases. Most intrinsic data is based upon analysis of a set of incremental couples prepared in the alloy system with a single marker at the original interface. The marker displacement, the Kirkendall shift, is reported for each couple. The simulation may be used with $D_{1}$ and $D_{2}$ as input to predict composition distributions and Kirkendall shifts in these couples. Figure 9 shows comparisons with these experimental
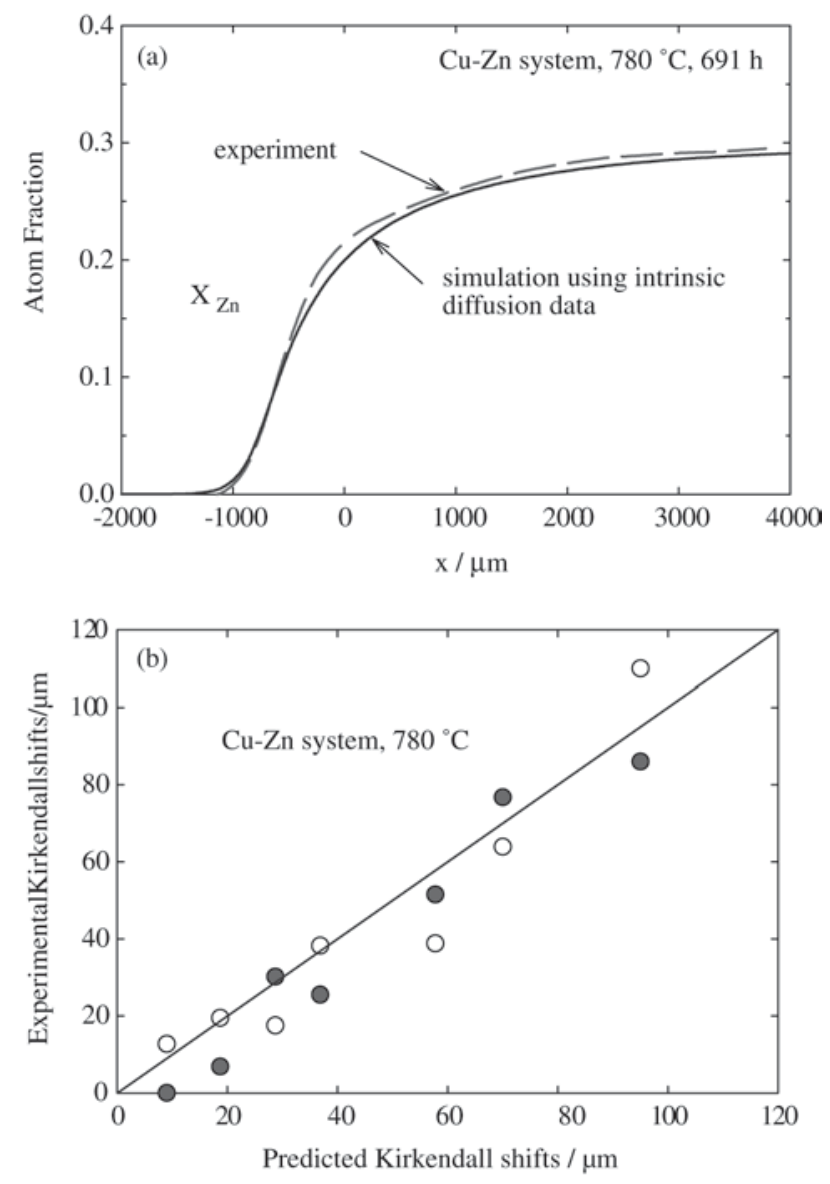

experiment $v s$. predicted data points
experiment including "estimated measurement error"
$v s$. predicted data points

Figure 9. a) Composition profile and b) Kirkendall shifts for the $\mathrm{Cu}-\mathrm{Zn}$ system ${ }^{32}$ obtained using the experimentally determined intrinsic diffusion coefficients as inputs to the diffusion simulation software. observables for the $\mathrm{Cu}-\mathrm{Zn}$ system. The consistency of the underlying procedures, the simulation and its predictions are clearly demonstrated in this comparison.

The simulation can be used to assess the connection between Darken's hypothesis and the experimental observables. Figs. 6 and 7 showed that the thermodynamic correction of tracer data proposed by Darken does not successfully describe the difference between tracer and intrinsic diffusivities. Figure 10 shows the correlation between measured composition distributions and Kirkendall shifts and values predicted by Darken's evaluation of the intrinsic diffusivities from tracer data. Kirkendall shifts predicted by Darken's equation are generally smaller than the measured shifts providing, another illustration of the inadequacy of Darken's hypothesis.
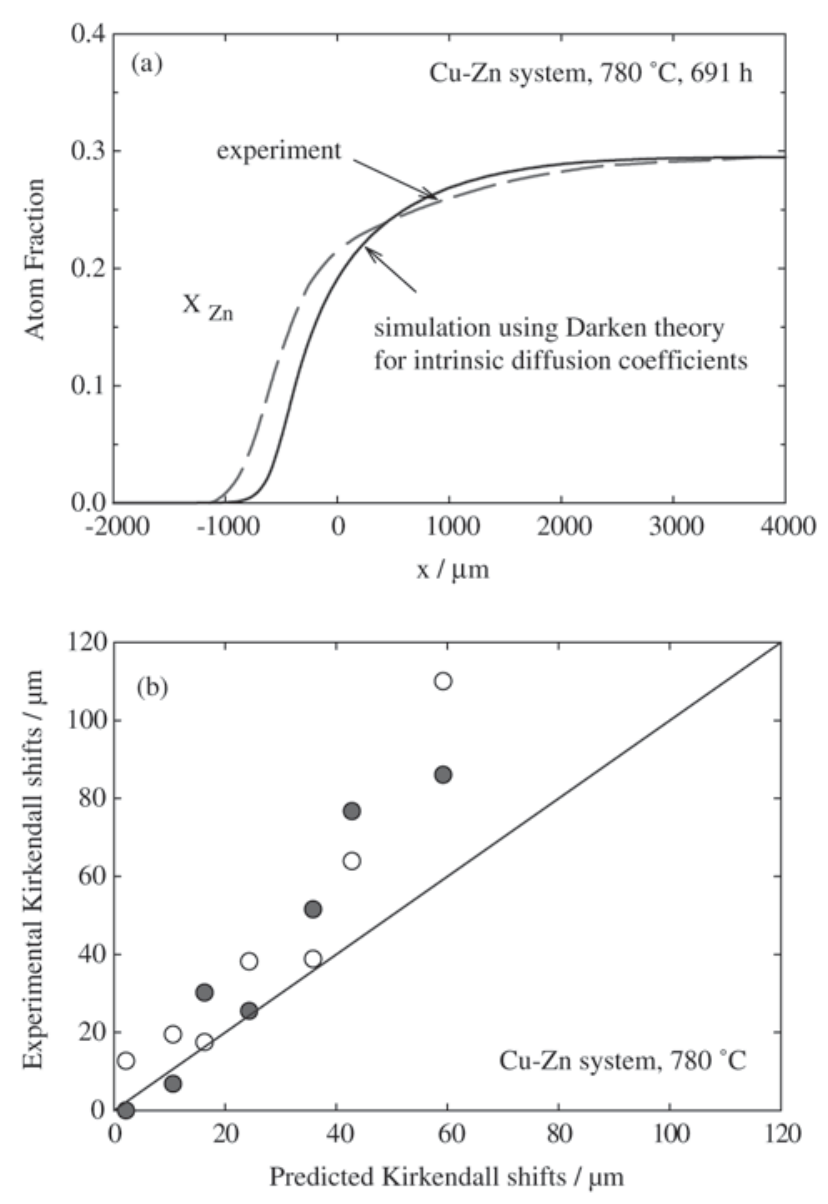

experiment $v s$. predicted data points

○ experiment including "estimated measurement error" $v s$. predicted data points

Figure 10. a) Concentration profile and b) Kirkendall shifts using the intrinsic diffusion coefficients obtained from the Darken theory ${ }^{8,9}$ as input to the simulation. 
The distributed marker experiment has not been undertaken for any ternary system. Intrinsic behavior has been probed in a few ternary systems ${ }^{24,41,42}$, using classical single marker couples. The simulation could be used in an iterative approach that would seek a set of the six diffusivity functions that would give consistent predictions of the observed anecdotal Kirkendall marker shifts. Such a program would require a significant level of effort and has not been undertaken. In concert with tracer data that exists in $\mathrm{Cu}-\mathrm{Ni}$ $\mathrm{Zn}$, the results of this exercise would provide a basis for the first test of the application of the Darken-Manning formalism to a ternary system.

\section{Simulation of the Kinetic Formalism for Intrinsic Diffusion}

The simulation of the kinetic formalism for intrinsic diffusion follows the procedure described above for the phenomenological intrinsic formalism. The only difference is the use of a finite difference form for the flux equation (33) to compute the flows at the boundaries of each volume element. Direct application of this equation using an experimentally derived data base is not yet possible because the content of the biased term is unknown. In view of this difficulty the simulation may be used in two ways:

1. The pattern of behavior of the unbiased term in the flux equation may be explored experimentally by combining intrinsic and tracer diffusion data to gain insight into the nature of the influences that operate to produce this bias.

2. A simplified version, neglecting the biased term, may be applied to the description of patterns of behavior in ternary systems.

These two applications are described briefly below.

A tracer diffusion experiment is a limiting case in which the fluxes of the components approach zero. Since the biased effects arise from the directed flow of the components, the biased effects decrease as the fluxes of the atoms decrease. Thus, in the limiting case of a tracer experiment the flux of tracer may be described by the unbiased term:

$$
J_{k}^{*}=J_{k}^{* U}=-\frac{1}{6} \lambda^{2} \frac{\partial C_{k}^{*} \Gamma_{k}^{*}}{\partial x}
$$

A central hypothesis in the development is that the jump frequencies depend only on composition and temperature, and not on the magnitudes of the flows in the system. Since the tracer experiment is a limiting case of a diffusion experiment as the driving forces and fluxes approach zero, the jump frequency in that limiting case at a given composition must be the same as that under larger driving forces. It is concluded that in the system with any set of magnitudes for the driving forces

$$
\Gamma_{\mathrm{k}}=\Gamma_{\mathrm{k}}{ }^{*}
$$

That is, the jump frequencies in the kinetic formalism are the tracer jump frequencies. Equation (33) may be written

$J_{k}=J_{k}^{B}+J_{k}^{U}=2 \alpha_{k} \lambda C_{k} \Gamma_{k}^{*}-\frac{1}{6} \lambda^{2} \frac{\partial C_{k} \Gamma_{k}^{*}}{\partial x}$

It has been shown that the total intrinsic flux, $J_{\mathrm{k}}$, can be evaluated without simplifying assumptions from the intrinsic diffusivities, or, more directly from the experimental evaluation of the interdiffusion fluxes and lattice velocity distribution. The unbiased term may be evaluated in the simulation from tracer measurements of the jump frequencies. The biased term may then be evaluated from this experimental information by subtracting the unbiased term from the total flux. Thus with the input of the tracer and intrinsic diffusion coefficients, the behavior of the biased contribution to the flux, $J_{\mathrm{k}}^{\mathrm{B}}$, can be explored in the system. Since the other factors in the expression for $J_{\mathrm{k}}{ }^{\mathrm{B}}$ are known, this permits a direct focus on the experimental determination of the content of the biased factor, $\alpha_{k+}$.

Total, biased and unbiased components of the intrinsic flux for a couple in the $\mathrm{Cu}-\mathrm{Zn}$ system, computed by implementing the simulation to process the data base information, are shown in Fig. 11. It is clear that the biased component of the flux cannot be neglected in describing the behavior of the system: $J_{\mathrm{k}}$ B is a significant fraction of $J_{\mathrm{k}}$ throughout the diffusion zone. It is further interesting that the signs of the biased factors for the two components, $\alpha_{1+}$ and $\alpha_{2+}$, are opposite in this case, Fig. 12. This observation implies that the bias cannot be explained simply as an effect due to the directed total flow of atoms or the corresponding vacancy flow. Other factors, still to be identified, appear to be involved.

The decomposition of the intrinsic flux into a biased and unbiased component provided by the kinetic formalism has the potential to provide a new level of insight into the physical influences involved in diffusion in multicomponent systems. These effects are just beginning to be explored.

In contrast with this rigorous analysis it must be noted that some success has been attained in describing patterns of diffusion behavior in ternary systems by using a simplified version of the kinetic flux equation in which the biased term is neglected. Figure 13 demonstrates that this is a bad assumption in the rigorous treatment of even binary systems if the jump frequencies are computed from tracer data. It is apparent from Fig. 13b that the predicted Kirkendall shifts are significantly higher than the experimental ones. If the requirement that the input jump frequencies are the tracer jump frequencies, is relaxed so that the input jump frequency surfaces over the Gibbs triangle are allowed to be modeled unconstrained by tracer information, then models have been 

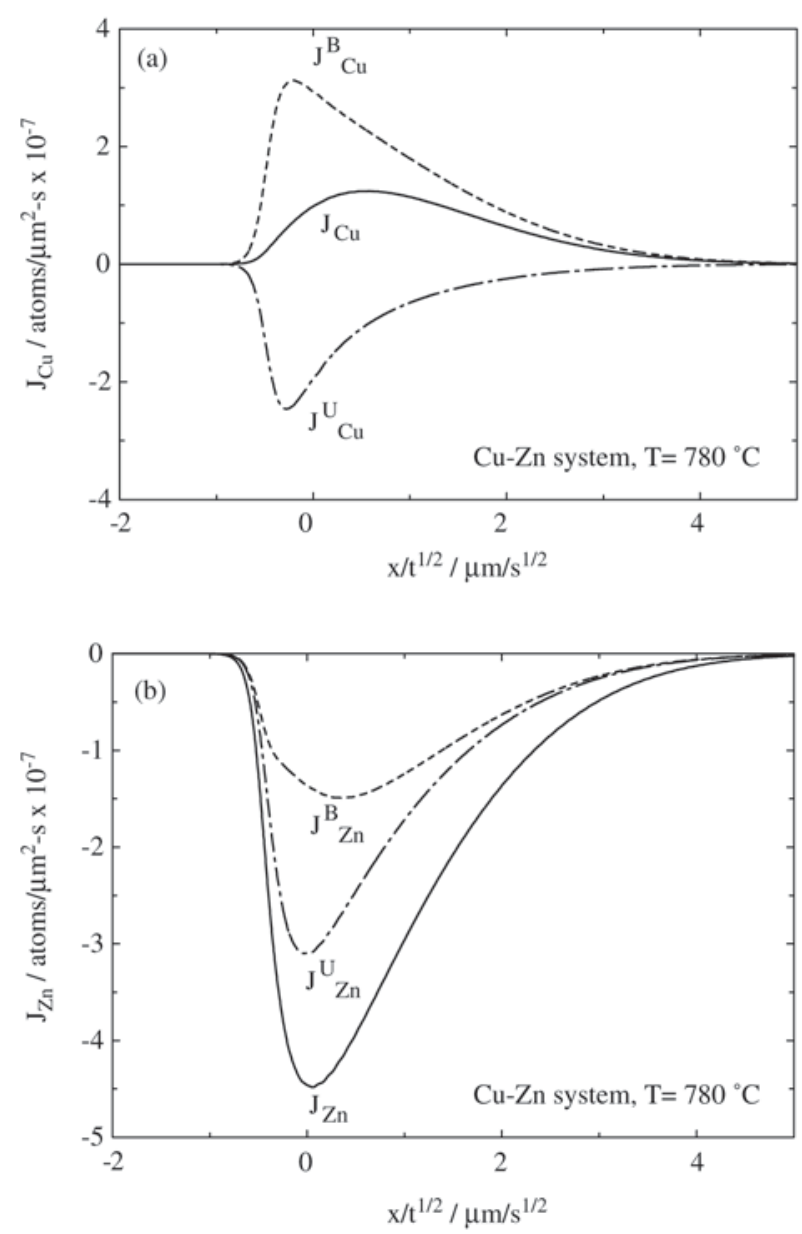

Figure 11. Total, biased and unbiased components of the intrinsic flux for a diffusion couple in the $\mathrm{Cu}-\mathrm{Zn}$ system $^{32}$. a) $\mathrm{Cu}$; b) $\mathrm{Zn}$.

devised that provide a reasonable explanation of most of the experimental observables in some ternary systems.

It is unlikely that full information about the pattern of tracer diffusion coefficients will be explored in ternary systems other than $\mathrm{Cu}-\mathrm{Ni}-\mathrm{Zn}$. This model approach sacrifices the rigor of the full kinetic equation for a practical version that can be made to work. If the biased term is neglected, equation (33) becomes

$$
J_{k}=-\frac{1}{6} \lambda^{2} \frac{\partial C_{k} \Gamma_{k}}{\partial x}
$$

Given a set of functions describing the composition dependence of the jump frequencies, $\Gamma_{k}\left(X_{k}\right)$, the simulation may be used to compute intrinsic fluxes as a function of position for any assumed initial couple. The experimental
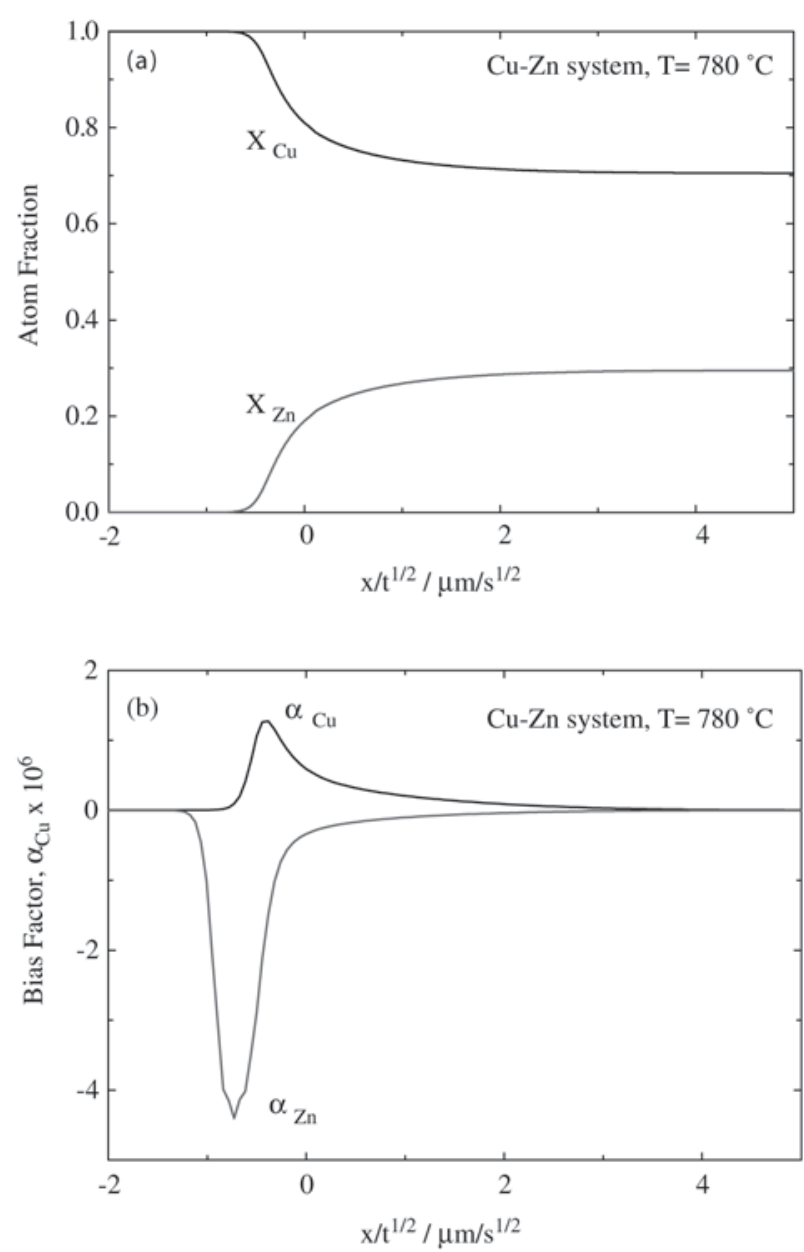

Figure 12. a) Composition profiles for the $\mathrm{Cu}-\mathrm{Zn}$ system and b) bias factors obtained from the biased flux shown in Fig. 11.

observables, i.e., composition distributions, composition paths and Kirkendall shifts, may be evaluated from this information and compared with observed patterns.

These jump frequency functions may be developed on the basis of an informed trial-and-error iteration by comparing patterns of composition paths with those obtained for a collection of such models ${ }^{44}$. Alternatively, jump frequency functions may be obtained by inverting equation (39) and integrating the result:

$$
\begin{gathered}
d\left(C_{k} \Gamma_{k}\right)=-\frac{6}{\lambda^{2}} J_{k}(x) d x \\
{\left[C_{k} \Gamma_{k}(x)\right]-\left[C_{k-} \Gamma_{k-}\right]=-\frac{6}{\lambda^{2}} \int_{x_{-}}^{x} J_{k}(x) d x}
\end{gathered}
$$



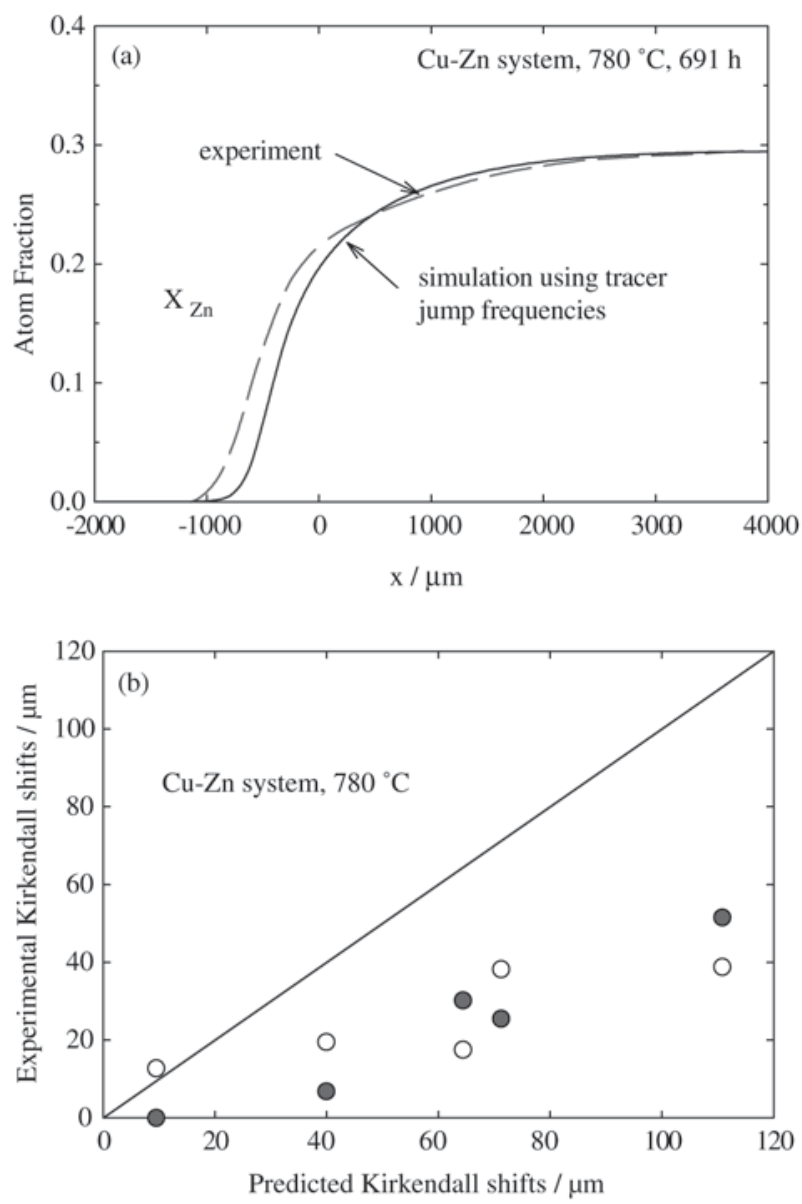

- experiment $v s$. predicted data points

○ experiment including "estimated measurement error" $v s$. predicted data points

Figure 13. a) Concentration profile; b) Kirkendall shifts using the tracer jump frequencies ${ }^{33}$ as inputs to the unbiased version of the jump frequency simulation.

The integrand is the intrinsic flux of component $\mathrm{k}$ determined experimentally from a distributed marker experiment. The second term on the left side of this equation is the value of the $C_{\mathrm{k}} \Gamma_{\mathrm{k}}$ product at the left end of the couple. If $C_{\mathrm{k}-}$ is chosen to be zero, or if $\Gamma_{\mathrm{k}-}$ has been determined previously, $\Gamma_{\mathrm{k}}$ can be determine as a function of composition for the set of compositions that exist in the couple.

Figure 14 compares the pattern of composition paths derived from applying the simulation (a) with that observed experimentally (b) for the Fe-Ni-Co system using the simple jump frequency model give in (c). All of the simulated composition paths have the same shape as the experimentally observed ones. In most cases the computed compositions are within a few atomic per cent of the experimental ones.
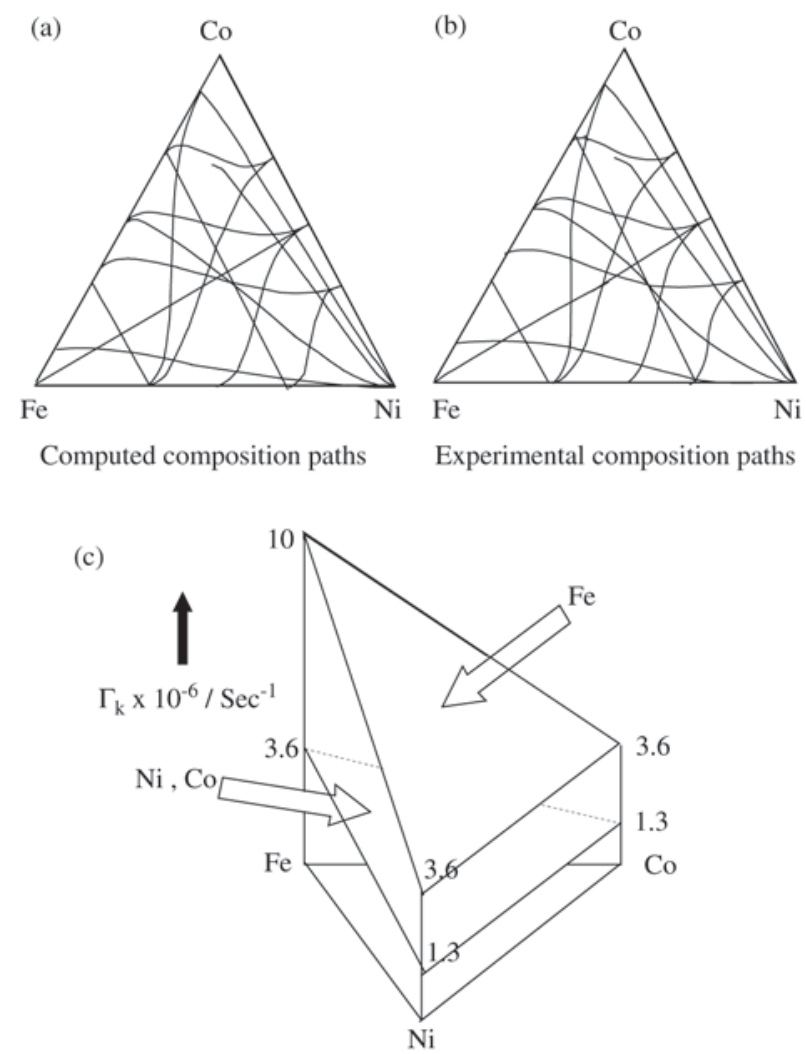

Figure 14. Comparison: a) simulated; b) experimental composition paths in the $\mathrm{Fe}-\mathrm{Co}-\mathrm{Ni}$ system ${ }^{43,44}$; c) The jump frequency model employed for this simulation ${ }^{44}$.

Figure 15 shows a more challenging test of this approach applied to the $\mathrm{Cu}-\mathrm{Ni}-\mathrm{Zn}$ system. In this case the jump frequency model, suggested by the pattern observed for tracer diffusion, has a very strong dependence upon composition. Further, the jump frequencies of the three components at a given composition are very different, maintaining a ratio of about $\mathrm{Zn}: \mathrm{Cu}: \mathrm{Ni}=9: 3: 1$. The composition paths have large amplitudes. Nonetheless the modeled paths all have the correct qualitative shape, though there are significant quantitative differences for couples with selected diffusion vectors on the Gibbs triangle. Further, Fig. 16 shows that the pattern of Kirkendall shifts is in general predicted reasonably well by the model through the simulation with the exception of those couples that also showed differences in the composition paths (Fig. 15a). Further improvement might be achieved through additional iterations of the modeled jump frequency surfaces.

This approach can also be applied to devise a model to explain the behavior observed in the Fe-C-Si. This text book example for "uphill diffusion", due to Darken, ${ }^{47}$ examines 

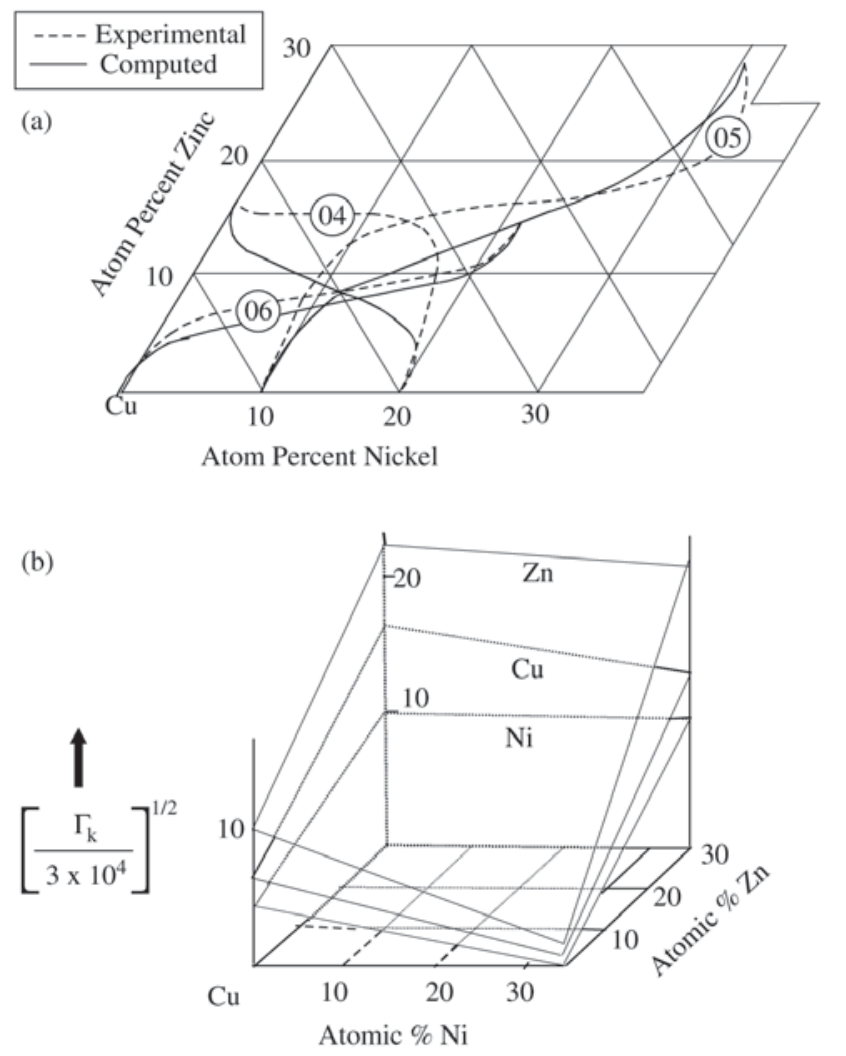

Figure 15. a) Comparison of experimental and simulated composition paths in th $\mathrm{Cu}-\mathrm{Ni}-\mathrm{Zn}$ system $^{44-46}$; b) The jump frequency model employed for this simulation ${ }^{44,46}$.

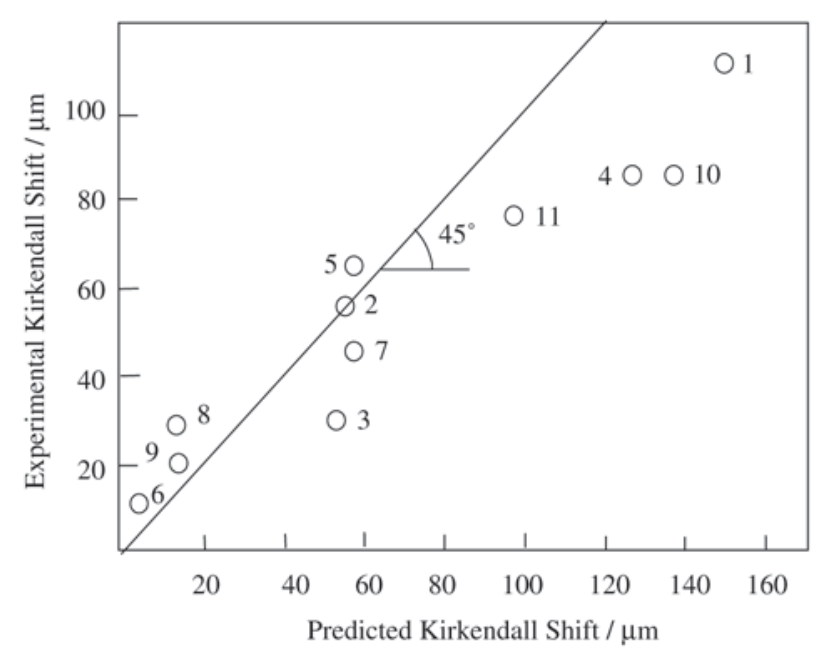

Figure 16. Comparison between the experimental and predicted Kirkendall shifts using the jump frequency model shown in Fig. 15 for various diffusion couples in the $\mathrm{Cu}-\mathrm{Ni}-\mathrm{Zn}$ system ${ }^{44,45}$. the composition profile produced in a couple that places an $\mathrm{Fe}-0.441 \% \mathrm{C}$ alloy against a ternary $\mathrm{Fe}-0.478 \mathrm{C}-3.8 \% \mathrm{Si}$ alloy. Although the carbon content is essentially the same on both sides of the couple it is found that carbon atoms significantly redistribute during diffusion, producing the profile shown in Fig. 17 (solid circles). Carbon flows out of the silicon rich side, where its concentration is reduced, and into the silicon free side where its concentration increases. Thus there is a range of composition for which the carbon atoms flow from low carbon content to high, up its concentration gradient. This observation was explained on the basis of the effect of silicon additions on the chemical potential of carbon in this system. The activity of carbon increases with silicon content. Thus the flow of carbon from the high silicon side of this ternary couple to the low silicon side is a flow down the chemical potential gradient of carbon, consistent with the mobility form of the phenomenological equations.

This behavior can also be reproduced by constructing a jump frequency model in which the jump frequency of carbon atoms increases with silicon content, and applying equation (39). Figure 17 shows the composition profile produced by this approach. This more physical explanation of the observation immediately invites additional question such as, "Why does the presence of silicon increase the jump frequency of carbon atoms?"

This modeling approach results in a workable description of the experimentally observed pattern of intrinsic dif-

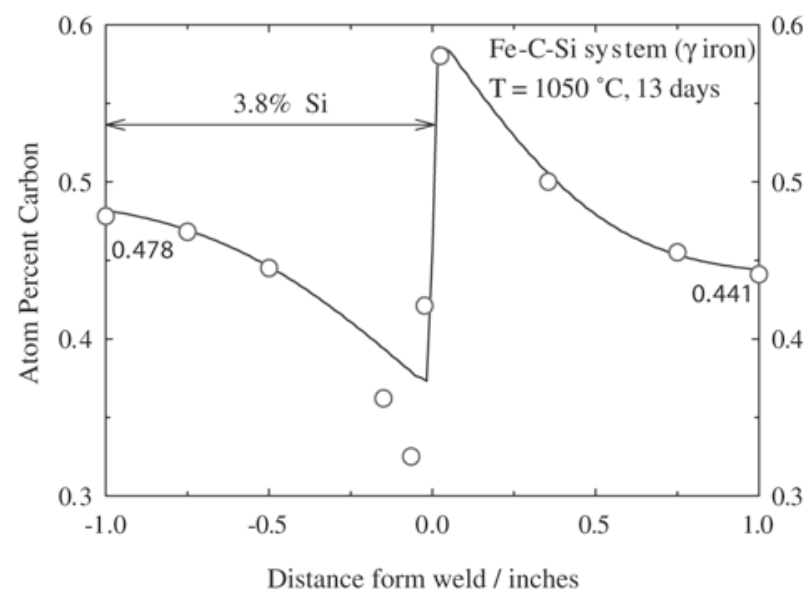

Figure 17. Simulation (solid line) of Darken's ${ }^{47}$ experiment demonstrating uphill diffusion of carbon in $\gamma$ iron at $1050{ }^{\circ} \mathrm{C}$ using a jump frequency model for carbon that is a function of the silicon concentration, $\Gamma_{\mathrm{C}}=\left(16 \mathrm{X}_{\mathrm{Si}}+1\right) 8 \times 109, \Gamma_{\mathrm{Fe}}=\Gamma_{\mathrm{Si}}=10^{6} \mathrm{sec}^{-1}$. The open circles represent concentrations from Darken's experiment. The simulated concentration profile was obtained by multiplying the normalized concentration profile (512 iterations) with the square root of the annealing time (13 days). 
fusion in a ternary system on the basis of a formalism that has physical meaning. The data base is a set of jump frequency values and their composition and temperature dependence. The operation of the governing equation (39) is easy to visualize. The approach can be extended easily to quaternary and higher order systems.

The trouble is, in order to invoke this approach it is necessary to ignore the results of the more rigorous analysis that recognizes that the jump frequencies are those obtained from tracer experiments, and that a significant component of the intrinsic flux derives from a physical bias in the jump directions of the components with respect to the flow direction. Implementation of the formalism without assumptions provides a new opportunity to explore the patterns of behavior of the biased contribution to the intrinsic flux and perhaps gain a new level of insight into the influences that are important in the diffusion process. Implementation of the formalism with empirically derived jump frequency models provides a practical avenue for predicting diffusion behavior in multicomponent systems.

\section{Discussion}

There exists a substantial and systematic data base for predicting diffusion in binary systems, but most useful alloys have more than two components. The data base for interdiffusion coefficients in ternary systems is very limited, and is practically nonexistent for systems with more then three components. This is mostly because the investment in experimental effort required to obtain these numbers is prohibitive, but partly because the values of the diffusivities in the data base don't make physical sense. The disconnect in the theory relating atom jump frequencies to interdiffusion coefficients means that there is no physical context for interpreting the values of the diffusivities. This lack of physical perspective in the interdiffusion data base in turn means that it is not possible to use these results to answer even rudimentary questions about the motions of the atoms, such as:

"Which components move fast in this system? Which are slow? What are their relative rates of motion? What are their absolute rates? How do these attributes change with composition in the system? What physical characteristics of the component are important in determining this behavior? What properties of the surrounding solution are important?"

Answers to questions like these could provide explanations of the observed diffusion behavior that might form the basis for interpolations and extrapolations within a system, and predictions of behavior in systems that have not been experimentally examined. These answers cannot be derived from interdiffusion information.
Use of distributed marker couples to obtain intrinsic fluxes and diffusivities does provide a path to this kind of information. However, preparation of samples with distributed markers, and their analysis, adds to an already prohibitive experimental effort. This technique has yet to be applied to a single ternary system. It seems unlikely that it will be adopted in the systematic study of multicomponent systems. The importance of intrinsic diffusion information in developing a physical understanding of diffusion in complex systems has not been widely appreciated. Because this approach provides independent information about the motions of the elements in the system it has the potential to provide the kind of insight, and eventually physical perspective, that can come from answers to the questions posed in the last paragraph.

Tracer diffusion information provides the most direct access to answers to the kinds of questions posed above. About a dozen tracer experiments are required to provide data on the composition dependence of $D_{1}^{*}$ and $D_{2} *$ in a binary system at a single temperature. This number escalates very rapidly with the number of components in the system. For example, to survey the three $D_{\mathrm{k}}{ }^{*}$ values for a ternary system over the Gibbs triangle, assuming the compositions chosen are 20 atomic per cent apart, requires 63 experiments. For a four component system sampling every $20 \%$ would require 224 diffusion coefficients. It is clear that the systematic survey of tracer diffusion behavior is not a practical route to a data base for multicomponent diffusion.

First principle calculations of atom jump frequencies are beginning to appear in the literature, but results are limited by the accuracy of the description of the atomic bonding models. This may be the wave of the future for developing diffusion data bases, but that future is very remote at this writing.

The kinetic flux equation, based as it is on jump frequencies of the components, has the potential for yielding answers to the questions posed early in this discussion. In its rigorous form, with both the biased and unbiased terms incorporated, this approach requires tracer data as input. Its systematic application to multicomponent systems thus requires the same prohibitive level of experimental effort inherent in tracer studies. Assessment of the biased term may provide new insight into influences that operate in flowing systems, but that potential is unknown at this writing.

Use of the simplified jump frequency flux equation to develop by iteration models of jump frequency functions that satisfactorily reproduce the experimental observables seems to emerge as the most practical approach to developing fundamental diffusion information for systems for which the number of components is greater than 2. For ternary systems best fit jump frequency functions for the three bi- 
nary systems provides the borders of the jump frequency surface over the Gibbs triangle. Distributed marker couples could be used to determine the intrinsic fluxes of all three components for a few couples that span the Gibbs triangle. Jump frequencies could then be computed along the composition paths for those couples by applying equation (40). Optimized jump frequency surfaces could then be developed from this binary and ternary information. The simulation could then be used to compare observables computed from this model with experimental observables. The result could then be tested with information for an additional couple or two to assess the predictions of the resulting model.

\section{Summary}

The experimental observables in a single phase multicomponent diffusion couple experiments are tracer penetration curves, marker displacement distributions and concentration profiles. Phenomenological equations define matrices of diffusivities, $D_{\mathrm{k}}{ }^{*}$ for tracer diffusion, $D_{\mathrm{kj}}{ }^{\circ}$ for interdiffusion and $D_{\mathrm{kj}}$ for intrinsic diffusion, which may be extracted by processing the experimental observables in sets of couple experiments. These sets of diffusivity matrices constitute the data base for the description of diffusion behavior. With values for these properties for an alloy system and the aid of an appropriate simulation one can predict the set of experimental observables for any diffusion scenario in the alloy system, given its initial and boundary conditions.

Procedures exist for the determination of intrinsic and interdiffusion coefficients for two and three component systems. Values of these descriptors are inaccessible for systems with more than three components.

Attempts to infer the physical content of these descriptors by relating intrinsic and interdiffusion coefficients to tracer information were undertaken by Darken, Manning and others. Tests of these relationships in a number of binary systems have demonstrated that the underlying hypothesis, i.e., that the tracer mobility and the intrinsic mobility are equal, is inadequate to explain the observed differences between tracer and intrinsic diffusivities. The predictions of this theory have never been tested for ternary or higher order systems. Indeed, they cannot be tested for systems with more than three components because the diffusivity matrices are inaccessible.

It is concluded that the physical content of the descriptors of the phenomenological formalism remains unknown even in binary systems. This approach has not provided a physical context for the diffusion data base from which a physical understanding or scientific explanation of patterns of observed behavior in multicomponent diffusion can be gleaned.

A version of the kinetic description of diffusion is pre- sented which computes the intrinsic flux from the composition dependent jump frequencies of the components. The development yields a biased and an unbiased contribution to the intrinsic flux.

The biased term is determined by influences that produce a difference in the fraction of jumps of an atom of a component in the plus and minus directions relative to the local flux. The biased term can be evaluated experimentally by combining tracer information and an oblique marker plane diffusion couple experiment, which permits experimental evaluation of the intrinsic flux of each component. Applications to binary system information from the literature show that the biased term cannot be neglected. The nature of the influences that produce this bias, and how these influences operate, is not yet known.

The unbiased term can be computed from tracer diffusion determinations of the composition dependence of the jump frequencies.

Although the biased term is important and complex, it has been found that reasonable descriptions of the experimental observables can be obtained if the constraint that the jump frequencies are determined from tracer data is relaxed and the biased term neglected. Models based upon heuristically derived jump frequency functions have been shown to yield semiquantitative descriptions of patterns of composition paths and Kirkendall shifts in some ternary systems.

Comparisons of the various formalism with their attendant hypotheses is made possible through the application of a flexible computer simulation of diffusion processes. This tool uses any of the diffusivity data bases as input and, by calculating the local flux at each position through a couple, computes the corresponding experimental observables.

The trouble with diffusion is that after more than half a century of experimental and theoretical investigation by some of the best minds in the material science field the prediction of the results of the operation of this crucial process remains based upon the phenomenological formalism which defines diffusivities that

- require significant experimental investment for their evaluation;

- are experimentally inaccessible for systems with more than three components;

- have unknown physical meaning.

Theoretical attempts to inject physical meaning into these properties by connecting intrinsic and interdiffusion phenomena to tracer diffusion behavior in the same system have been shown to be inadequate. An alternative path to the physical understanding of the diffusion process leads to a kinetically based flux equation in which all of the parameters have a known physical meaning except a biased factor. The trouble in this case is: the content of the biased factor is unknown. 


\section{References}

1. Haase, R. Thermodynamics of Irreversible Processes, Addison Wesley, Reading, MA, 1969.

2. Kirkaldy, J.S.; Young, D.J. Diffusion in the Condensed State, Institute of Metals, London, 1987.

3. Philibert, J. Atom Movements: Diffusion and Mass Transport in Solids, translated by S.J. Rothman, Les Editions de Physique, Cedex, France, 1991.

4. Shewmon, P. Diffusion in Solids, Second Edition, TMS, Warrendale, PA, 1989.

5. Dayananda, M.A.; Kim, C.W. Met. Trans. A, v. 10, p. 1333-1339, 1979.

6. Kim, C.W.; Dayananda, M.A. Met. Trans. A, v. 14, p. 857-864, 1983.

7. Smigelskas, A.D.; Kirkendall, E.O. Trans. AIME, v. 171, p. 130-142, 1947.

8. Darken, L.S. Trans. AIME, v. 175, p. 184-201, 1948.

9. Darken, L.S. Atom Movements, ASM, Cleveland, OH, p. $1-25,1950$

10. Cornet, J.F.; Calais, D. J. Phys. Chem. Solids, v. 33, p. 1675-1684, 1972.

11. Cornet, J.F. J. Phys. Chem. Solids, v. 35, p. 1247-1252, 1974.

12. Kohn, A.; Levasseur, J.; Philibert, J.; Wanin, M. Acta. Met., v. 18, p. 163-173, 1970.

13. Van Dal, M.J.H.; Pleumeekers, M.C.L.P.; Kodentsov, A.; Van Loo, F.J.J. Acta. Met., v. 48, p. 385-396, 2000.

14. Van Dal, M.J.H.; Pleumeekers, M.C.L.P.; Kodentsov, A.; Van Loo, F.J.J. J. Alloys \& Comp., v. 309, p. 132140, 2000.

15. de Groot, S.R.; Mazur, P. Non-Equilibrium Thermodynamics, North Holland Press, Amsterdam, 1962.

16. Onsager, L. Phys. Rev., v. 37, p. 405-426, 1931; v.38, p. 2265-2279, 1932.

17. Allnatt, A.R.; Lidiard, Atomic Transport in Solids, Cambridge University Press, Cambridge, 1993.

18. DeHoff, R.T. Thermodynamics in Materials Science, McGraw-Hill, New York, 1993.

19. Gaskell, D.R. Introduction to Metallurgical Thermodynamics, Second Edition, Hemisphere Publishing, New York, 1981.

20. Lupis, C.H.P. Chemical Thermodynamics of Materials, p. 363-364, North Holland (Elsevier Science), New York, 1983.

21. Thompson, M.S.; Morral, J.E. Acta Met., v. 34, p. 339346, 1986.

22. Stalker, M.K.; Morral, J.E.; Romig Jr., A.D. Met. Trans. A, v. 23, p. 3245-3249, 1991.

23. Manning, J.R. Met. Trans., v. 1, p. 499-505, 1970.
24. Guy, A.G.; Philibert, J.; Z. Metallkde., v. 56, p. 841845, 1965.

25. Iijima, Y.; Hirano, K.; Kikuchi, M. Trans. Jap. Inst. Met., v. 23, p. 19-23, 1982.

26. Onishi, M.; Miyake, K.; Wakamatsu, Y.; Shimozaki, T. Defect and Diffusion Forum, v. 95-98, p. 561-572, 1993. Proceedings DIMAT 92, Diffusion in Materials, Kyoto, Japan, eds. M. Koiwa, K. Hirano, H. Nakajima and T. Okada, Trans. Tech Publ., 1993.

27. Heuman, T.; Rottwinkel, T. J. Nucl. Mat., v. 69 \& 70, p. 567-570, 1978.

28. Heuman, T. in Dimeta-82, Diffusion in Metals and Alloys, Proceedings, edited by F.J. Kedves and D.L. Beke, Debrecen, Hungary, Diffusion and Defect Monograph Series, n. 7, p. 117-133, 1983.

29. Iorio, N.R.; Dayananda, M.A.; Grace, R.E. Met. Trans. A, v. 4, p. 1339-1346, 1973.

30. Cheng, G.H.; Dayananda, M.A.; Grace, R.E. Met. Trans. A, v. 6, p. 21-27, 1975.

31. Schmatz, D.J.; Domain, H.A.; Aaronson, H.I. J. App. Phy., v. 37, p.1741-1743, 1966.

32. Horne, G.T.; Mehl, R.F. Trans. AIME, v. 203, p. 88-99, 1955.

33. Anusavice, K.J.; DeHoff, R.T. Met. Trans. A, v. 3, p. 1279-1298, 1972.

34. Kowalski, M.; Spencer, P.J. J. Phase Equil., v. 14, p. 432-438, 1993.

35. Filby, J.D.; Pratt, J.N. Acta. Met., v. 11, p. 427-434, 1963.

36. Manning, J.R. Phy. Rev., v. 139, A 126-135, 1965.

37. Manning, J.R. Acta Met., v. 15, p. 817-826, 1967.

38. J. R. Manning, Diffusion Kinetics of Atoms in Crystals, D. Van Norstrand, Princeton, 1968.

39. Manning, J.R. in Diffusion Analysis \& Applications, ed. A.D. Romig, Jr. and M.A. Dayananda, published by TMS. Proceedings of TMS Fall Meeting, Chicago, IL, p. 3-17, Sept. 1988.

40. Dayananda, M.A. Acta Met., v. 29, p. 1151-1157, 1981.

41. Carlson, P.T.; Dayananda, M.A.; Grace, R.E. Met. Trans. A, v. 3, p. 819-826, 1972.

42. Sisson Jr., R.D.; Dayananda, M.A. Met. Trans. A, v. 8, p. 1849-1856, 1977.

43. Vignes, A.; Sabatier, J.P. Trans. AIME, v. 245, p. 1795$1802,1969$.

44. Iswaran, C.V. Ph.D. Thesis, Univ. of Florida, Gainesville, 1993.

45. Wan, C.C. Ph. D. Thesis, Univ. of Florida, Gainesville, 1973.

46. Wan, C.C.; DeHoff, R.T. Acta Met., v. 25, p. 287-294, 1977. 